\title{
THE STRUCTURE OF LEGENDRE FOLIATIONS
}

\author{
MYUNG-YULL PANG
}

\begin{abstract}
The local and global structure of Legendre foliations of contact manifolds is analysed. The main invariant of a Legendre foliation is shown to be a quadratic form on the tangent bundle to the foliation-the fundamental quadratic form. The equivalence problem is solved in the case when the fundamental quadratic form is nondegenerate and a generalization of Chern's solution to the equivalence problem for Finsler manifolds is obtained. A normal form for Legendre foliations is given which is closely related to Weinstein's structure theorem for Lagrangian foliations. It is shown that every compact, simply connected leaf of a Legendre foliation is diffeomorphic to a sphere.
\end{abstract}

\section{INTRODUCTION}

Let $P$ be a smooth $(2 n+1)$-dimensional manifold without boundary with a fixed choice of contact 1-form $\eta$ (i.e. $\eta \wedge(d \eta)^{n} \neq 0$ ). A Legendre foliation is a foliation of $P$ by $n$-dimensional integral submanifolds of $\eta$. We say that two Legendre foliations $\mathscr{F}_{1}$ and $\mathscr{F}_{2}$ are equivalent if there exists a diffeomorphism $\phi: P_{1} \rightarrow P_{2}$ satisfying the conditions

$$
\phi^{*} \eta_{2}=\eta_{1} \quad \text { and } \quad \phi^{*} \mathscr{F}_{2}=\mathscr{F}_{1}
$$

where $\phi^{*} \mathscr{F}_{2}$ is the foliation of $P_{1}$ whose leaves are inverse images under $\phi$ of leaves of $\mathscr{F}_{2}$. The purpose of this paper is to study the geometry of Legendre foliations with particular emphasis on the problem of determining when two Legendre foliations are equivalent.

The geometry of Legendre foliations is closely related to the geometry of Lagrangian foliations of symplectic manifolds [W]. To understand the relation, begin by recalling that the cotangent space $T^{*} M$ of a smooth manifold $M^{n+1}$ has a tautological 1 -form $\zeta$ and that $T^{*} M$ is equipped with a natural symplectic structure defined by the 2 -form $-d \zeta$. The foliation by fibers of $\pi: T^{*} M \rightarrow$ $M$ is a Lagrangian foliation (i.e. the leaves are $(n+1)$-dimensional integral submanifolds of $-d \zeta)$. Let $F: T^{*} M \rightarrow \mathbb{R}^{+} \cup\{0\}$ be a function such that

$$
F(t v)=t F(v) \text { for all } t \geq 0 \text { and } v \in T^{*} M
$$

and set $S_{F}^{*} M=\left\{v \in T^{*} M \mid F(v)=1\right\}$. For example, if $F$ is the norm defined by a Riemannian metric $g$ on $M$, then $S_{F}^{*} M$ is the unit cotangent bundle $S_{g}^{*} M$

Received by the editors April 12, 1989.

1980 Mathematics Subject Classification (1985 Revision). Primary 53B40; Secondary 53C57. 
on $M$. The set $S_{F}^{*} M$ is a $(2 n+1)$-dimensional submanifold of $T^{*} M$ with contact 1-form defined by the pull back to $S_{F}^{*} M$ of $\zeta$ and Legendre foliation $\mathscr{F}_{F}$ defined by the fibers of the projection map. One of the main results in this paper is the following theorem:

Theorem. Any Legendre foliation $\mathscr{F}$ is locally equivalent to one of the form $\mathscr{F}_{F}$. This is an analogue of the following result of Weinstein [W]:

Theorem. Any Lagrangian foliation is locally equivalent to one of the form defined by fibers of $\pi: T^{*} M \rightarrow M$. Moreover, the leaves of the foliation have a natural flat affine structure.

To classify Legendre foliations, we introduce two local invariants: (i) the fundamental quadratic form $\Pi$ which is a symmetric 2 -form on the tangent bundle to $\mathscr{F}$, and (ii) the invariant $\mathbf{G}$, a symmetric 3 -form on the tangent bundle to $\mathscr{F}$. For Legendre foliations of the form $\mathscr{F}_{F}, \Pi$ and $\mathbf{G}$ are restrictions to the tangent bundle of $\mathscr{F}_{F}$ of the following symmetric forms:

$$
\boldsymbol{\Pi}=\frac{\partial^{2} F}{\partial y^{i} \partial y^{j}} d y^{i} \otimes d y^{j}, \quad \mathbf{G}=\frac{1}{2} \frac{\partial^{3} F}{\partial y^{i} \partial y^{j} \partial y^{k}} d y^{i} \otimes d y^{j} \otimes d y^{k} .
$$

The invariant $\mathbf{G}$ was originally introduced by Chern in the special case when $F$ is a Finsler metric, and he proved that $\mathbf{G}$ vanishes precisely when the Finsler metric is Riemannian, in which case $\Pi$ coincides with the induced metric on the fibers. The quantities $\boldsymbol{I}$ and $\mathbf{G}$ have an invariant definition in terms of Lie derivatives (Proposition 3.3)

The study of Legendre foliations in this paper is motivated by work with Duchamp and Uhlmann [DPU] on the inverse scattering problem for metrics: Let $g$ be a Riemannian metric on $\mathbb{R}^{n+1}$ different from the standard euclidean metric only on the interior of the ball of radius 1 . The inverse scattering problem is to prove the uniqueness (up to diffeomorphism) of the metric $g$ from the scattering data of geodesics (i.e. the behavior of geodesics entering and leaving the unit ball.) Presently, the uniqueness is known only in the special case when the metrics do not have conjugate points inside the ball and are conformally equivalent. The problem is still open under more general conditions. The inverse scattering problem for metrics can be reformulated in terms of Legendre foliations as follows. Let $\tilde{g}$ be a small deformation of the metric $g$ with $\tilde{g}=g$ outside of the ball and which has the same scattering data for geodesics as $g$. In [DPU] we show that there is a natural way to associate to $\tilde{g}$ a deformation $\widetilde{F}$ of the Legendre foliation $\mathscr{F}_{g}$ on $S_{g}^{*} \mathbb{R}^{n+1}$ with the properties that

(i) the equation $\widetilde{\mathbf{G}}=0$ holds where $\widetilde{\mathbf{G}}$ is the invariant tensor associated (ii) $\stackrel{\text { to }}{\widetilde{F}}$ coincic The relation to the inverse scattering problem can be easily seen from the following theorem [DPU]: 
There is an isometry $f:\left(\mathbb{R}^{n+1}, g\right) \rightarrow\left(\mathbb{R}^{n+1}, \tilde{g}\right)$ which is an identity outside the ball if and only if

$$
\widetilde{\mathscr{F}}=\mathscr{F}_{g} .
$$

Conditions (i) and (ii) can be interpreted as a boundary value problem in partial differential equations. We are presently working on proving uniqueness in the special case $n=1$ where the integrability conditions for the foliation are trivially satisfied. In higher dimensions, the integrability conditions for the distribution defining $\widetilde{F}$ must be incorporated and the best formulation of the system of P.D.E.'s is yet to be determined.

Legendre foliations with nondegenerate $\Pi$ are called nondegenerate. The study of the local structure of Legendre foliations is closely related to Chern's work on Finsler space [C]: We show that the condition that $\Pi$ is positive definite is equivalent to the condition that the Legendre foliation is locally equivalent to one of the form $\mathscr{F}_{F}$ with $F$ a Finsler metric (i.e. $F^{2}$ is a convex function on $T_{x}^{*} M$ for all $x \in M$ ).

The local structure of nondegenerate Legendre foliations is also related to the works of R. Gardner, R. Bryant and, more recently, of N. Kamran and P. Olver on Calculus of Variations. For instance, in the special case when $n=1$, the structure equations of a nondegenerate Legendre foliation turn out to be the same as those of a nondegenerate first order Lagrangian [G]. The equivalence problem for first order Lagrangians is discussed in more detail in [ $\mathrm{Br}$ and $\mathrm{KO}$ ].

The paper is organized as follows. $\S 1$ is an introduction to basic definitions and examples of Legendre foliations. We prove the normal form theorem in $\S 2$. In $\S 3$, the local invariants $\Pi$ and $\mathbf{G}$ and their invariant definitions are introduced.

In $\S 4$, we describe the simplest class of Legendre foliations characterized by the vanishing of $\Pi$. Such Legendre foliations are called flat. We show that any flat Legendre foliation is locally equivalent to one defined on $S_{F}^{*} M$ with $F=y^{n+1}$. In particular, all the flat Legendre foliations are equivalent and the leaves possess a natural flat affine structure.

$\S 5$ is the local study of Legendre foliations with nondegenerate $\Pi$. This is accomplished by showing that there is a natural reduction of the structure group of $P$ to the orthogonal group $O(p, q)$ where $(p, q)$ is the signature of the fundamental quadratic form. By suitably normalizing the torsion, we obtain a canonical connection on $P$. Its torsion and the curvature form a set of local invariants.

One result of the reduction is that a CR-structure can be defined on $P$ in a natural way. In general, this CR-structure is not integrable (i.e. the holomorphic tangent bundle on $P$ is not closed under the Lie bracket [LB]). However, we show that the CR-structure obtained from the Legendre foliation on the unit cotangent bundle of a Riemannian manifold with constant sectional curvature is integrable. In the special case when the dimension of $P$ is 5 and $\Pi$ is positive definite, there is another CR-structure on $P$. We give necessary and sufficient 
condition for the integrability of this CR-structure in terms of the invatiant $\mathbf{G}$ (Proposition 5.45). In the special case when $\mathbf{G}=0$, the integrability condition is trivially satisfied, and the CR-structure is locally equivalent to the one defined by LeBrun [LB].

In $\S 6$, we study the global structure of Legendre foliations. We prove that every compact and simply connected leaf of a Legendre foliation is diffeomorphic to an $n$-sphere. More generally, it can be shown that every compact leaf with finite fundamental group is diffeomorphic to a quotient of a sphere. From the theory of foliations, it can be shown that if all leaves of a foliation $\mathscr{F}$ are simply connected and compact, then the leaf space $P / \mathscr{F}$ is a manifold. Under this assumption, the normal form theorem generalizes to a global theorem:

Theorem. A Legendre foliation with all of its leaves compact and simply connected is globally equivalent to one of the form $\mathscr{F}_{F}$ defined on $S_{F}^{*}(P / \mathscr{F})$, and the fibers are all diffeomorphic to $n$-spheres. If in addition $\Pi$ is nondegenerate, then $F$ is a Finsler metric.

It follows from the local theory that in the special case where $\Pi$ is positive definite and $\mathbf{G}$ vanishes the leaves of $\mathscr{F}$ have a natural metric of positive constant sectional curvature. Consequently, each compact leaf is diffeomorphic to a quotient of a sphere by a finite group. Moreover, if $P$ is compact, all the leaves are necessarily compact, and hence diffeomorphic to quotients of spheres.

Notation. Throughout this paper, the following notations are used.

(i) The summation convention is used: The latin indices $i, j, \ldots$ range from 1 to $n+1$, and the greek indices from 1 to $n$.

(ii) All the manifolds are smooth and without boundary. If $B \rightarrow P$ is a vector bundle, $\Gamma(B)$ denotes the set of all smooth sections of $B$. Functions and sections of vector bundles are smooth.

(iii) $i(X) \omega$ denotes the interior evaluation of a vector field $X$ on a differential form $\omega$.

(iv) $\left(x^{i}, y^{j}\right)$ denote the coordinates on $T^{*} M$ induced from coordinates $\left(x^{i}\right)$ on an $(n+1)$-dimensional manifold $M$. If $F$ is a function on $T^{*} M$, then $F_{i}=\frac{\partial F}{\partial y^{i}}, F_{i j}=\frac{\partial^{2} F}{\partial y^{i} \partial y^{j}}$, and so on.

I would like to thank Professor T. Duchamp for his guidance and many suggestions which were very helpful throughout the paper, especially for the global results. I am also grateful to Professor J. Lee for his suggestion to consider the relation to CR-geometry. The work in this paper was submitted as part of the author's doctoral thesis at the University of Washington in 1989.

\section{Definitions AND EXAMPLES}

1.1 Legendre foliations. A contact distribution $H$ on $P$ is a $2 n$-dimensional subbundle of $T P$ which has the property that any local trivialization $\eta$ of the line bundle $E^{*}=\operatorname{Ann}(H) \subset T^{*} P$ (i.e. nonzero section of $E_{\mid U}^{*} \rightarrow U$ defined on a neighborhood $U \subset P$ ) satisfies the condition $\eta \wedge(d \eta)^{n} \neq 0$. We call a pair $(P, H)$ a contact manifold in the wider sense [B]. 
If $E^{*}$ is trivial as a vector bundle, $E^{*}$ has a globally defined nonzero section $\eta$. We call $\eta$ a contact 1 -form and a contact manifold is defined to be a pair $(P, \eta)$. Clearly, a contact manifold is also a contact manifold in the wider sense with the contact distribution defined by $\operatorname{Ann}(\eta)$. The contact 1 -form determines a vector field $\mathbf{X}_{\eta}$ on $P$, called the characteristic vector field, by the conditions $i\left(\mathbf{X}_{\eta}\right) \eta=1$ and $i\left(\mathbf{X}_{\eta}\right) d \eta=0$. The charateristic vector field gives a natural decomposition $T^{*} P=E^{*} \oplus \operatorname{Ann}\left(\mathbf{X}_{\eta}\right)$.

1.2 Definition. A Legendre foliation (resp. in the wider sense) $\mathscr{F}$ of a contact manifold $(P, \eta)$ is a foliation by $n$-dimensional integral submanifolds of $\eta$ (resp. of $H$ ). Two Legendre foliations (resp. in the wider sense) $\mathscr{F}_{1}$ and $\mathscr{F}_{2}$ are said to be equivalent if there is a diffeomorphism $\phi: P_{1} \rightarrow P_{2}$ satisfying two conditions

$$
\phi^{*} \eta_{2}=\eta_{1}\left(\text { resp. } \phi^{*} E_{2}^{*}=E_{1}^{*}\right), \quad \phi^{*} \mathscr{F}_{2}=\mathscr{F}_{1},
$$

where $\phi^{*} \mathscr{F}_{2}$ is the foliation of $P_{1}$ whose leaves are the inverse images under $\phi$ of leaves of $\mathscr{F}_{2}$.

Given a foliation $\mathscr{F}$, the tangent bundle $\pi_{L}: L \rightarrow P$ to $\mathscr{F}$ is the subbundle of TP consisting of tangent vectors to the foliation. Similarly, the conormal bundle $\pi_{N}: N^{*} \rightarrow P$ to $\mathscr{F}$ is the subbundle of $T^{*} P$ consisting of covectors normal to $\mathscr{F}$. A tangential vector field is a section of $L$, and a relative 1-form is a section of $L^{*}$. In the case when $\mathscr{F}$ is a Legendre foliation with contact 1 -form $\eta$, the conormal bundle has a canonical splitting

$$
N^{*}=E^{*} \oplus Q^{*}
$$

where $\pi_{Q}: Q^{*} \rightarrow P$ is the subbundle of $N^{*}$ defined by $Q^{*}=N^{*} \cap \operatorname{Ann}\left(\mathbf{X}_{\eta}\right)$. Moreover, there is a natural vector bundle isomorphism $\Phi: L \rightarrow Q^{*}$ defined as follows. For $X \in L$, let $\Phi(X)=i(X) d \eta \in T^{*} P$. But, $i(X) d \eta \in \Gamma\left(Q^{*}\right)$ since $\{i(X) d \eta\}\left(\mathbf{X}_{\eta}\right)=0$ and $\{i(X) d \eta\}(Y)=-\eta([X, Y])=0$ for all $Y \in \Gamma(L)$. $\Phi$ is an injection because $d \eta$ is nondegenerate on $\operatorname{Ann}\left(\mathbf{X}_{\eta}\right)$ and, since $L, Q^{*}$ are of the same dimension, $\Phi$ is an isomorphism.

1.4 Projective cotangent bundles. An important class of examples of Legendre foliations comes from the projective cotangent bundles. Let $M$ be a $(n+1)$ dimensional manifold and $T_{0}^{*} M \subset T^{*} M$ the set of nonzero covectors. The projective cotangent bundle $\pi_{P}: \mathbb{P}_{+}^{*} M \rightarrow M$ is a fiber bundle defined as the quotient of $T_{0}^{*} M$ under the action of positive real numbers $\mathbb{R}^{+}$by multiplication on $T_{0}^{*} M$.

To define the contact distribution $\mathbb{P}_{+}^{*} M$, we make use of the the canonical 1-form $\zeta$ on $T^{*} M$ defined by the formula

$$
\zeta_{v}(X)=v\left(\pi_{*} X\right)
$$

where $v \in T^{*} M, X \in T_{v}\left(T^{*} M\right)$, and $\pi: T^{*} M \rightarrow M$ is the natural projection. 
The form $\zeta$ has the local expression

$$
\zeta=\sum_{i=1}^{+1} y^{i} d x^{i}
$$

where $\left(x^{i}, y^{j}\right)$ are the coordinates on $T^{*} M$ induced by coordinates $\left(x^{i}\right)$ on $M$. It is well known that the 2 -form $-d \zeta=\sum_{i=1}^{n+1} d x^{i} \wedge d y^{i}$ defines a symplectic structure on $T^{*} M$.

We define a contact 1-form on $\mathbb{P}_{+}^{*} M$ as follows. Let $F: T^{*} M \rightarrow \mathbb{R}$ be a function satisfying the homogeneity condition

$$
F(t v)=t F(v) \quad \text { for } v \in T^{*} M \text { and } t \geq 0 .
$$

For example, $F$ can be the induced norm on the cotangent bundle of $M$ associated to a Riemannian metric $g$. If $F$ is positive on $T_{0}^{*} M, \frac{\zeta}{F}$ is a well-defined 1-form of homogeneous degree zero on $T_{0}^{*} M$, and hence it remains invariant under the multiplicative action of $\mathbb{R}^{+}$. The 1 -form $\frac{\zeta}{F}$ defines the contact 1 -form $\eta_{F}$ on the quotient manifold $\mathbb{P}_{+}^{*} M$.

To see that $\eta_{F}$ is a contact 1 -form on $\mathbb{P}_{+}^{*} M$ (i.e. $\eta_{F} \wedge\left(d \eta_{F}\right)^{n} \neq 0$ ), proceed as follows: Let $\pi_{+}: T_{0}^{*} M \rightarrow \mathbb{P}_{+}^{*} M$ be the natural projection map. Then $\pi_{+}$is a bundle map over $P$ such that $\pi_{+}^{*} \eta_{F}=\frac{\zeta}{F}$. Then the condition $\eta_{F} \wedge\left(d \eta_{F}\right)^{n} \neq 0$ follows from the following computation:

$$
\begin{aligned}
\pi_{+}^{*}\left\{\eta_{F} \wedge\left(d \eta_{F}\right)^{n}\right\} & =\frac{\zeta}{F} \wedge\left\{d\left(\frac{\zeta}{F}\right)\right\}^{n}=\frac{\zeta}{F} \wedge\left\{-\frac{1}{F^{2}} d F \wedge \zeta+\frac{d \zeta}{F}\right\}^{n} \\
& =\frac{1}{F^{n+1}} \zeta \wedge(d \zeta)^{n} \neq 0 .
\end{aligned}
$$

It is clear that a different choice of $F$ will define the same contact distribution on $\mathbb{P}_{+}^{*} M$ since it will at most differ by a conformal factor (i.e. if $F^{\prime}$ is a different choice, $\eta_{F}=f \eta_{F^{\prime}}$ for some $\left.f \in C^{\infty}\left(\mathbb{P}_{+}^{*} M\right)\right)$, and hence the contact distribution defined by $\eta_{F}$ is canonical (i.e. independent of choice of $F$ ). On $\mathbb{P}_{+}^{*} M$, a Legendre foliation in the wider sense is defined by the fibers of $\pi_{P}: \mathbb{P}_{+}^{*} M \rightarrow M$.

1.7 Unit cotangent bundles. It is clear that a fixed choice of $F$ gives a Legendre foliation on $\mathbb{P}_{+}^{*} M$, denoted by $\mathscr{F}_{F}$. The Legendre foliation $\mathscr{F}_{F}$ can be equivalently described as follows: We call the manifold $S_{F}^{*} M=F^{-1}(1) \subset T^{*} M$ the unit cotangent bundle. $S_{F}^{*} M$ is a contact manifold with contact 1-form $\eta_{F}$ given by pulling back of the canonical 1 -form $\zeta$; and the foliation of $S_{F}^{*} M$ given by the fibers of the projection map $S_{F}^{*} M \rightarrow M$ defines the Legendre foliation equivalent to $\mathscr{F}_{F}$. The equivalence can be easily seen from the diffeomorphism obtained by composing the following maps:

$$
S_{F}^{*} M \hookrightarrow T^{*} M \stackrel{\pi_{+}}{\longrightarrow} \mathbb{P}_{+}^{*} M .
$$

On $S_{F}^{*} M$, the characteristic vector field $\mathbf{X}_{\eta}$ can be calculated directly by using 
the induced coordinates on $T^{*} M$ :

$$
\mathbf{x}_{\eta}=\sum_{i=1}^{n+1}\left\{\frac{\partial F}{\partial y^{i}} \frac{\partial}{\partial x^{i}}-\frac{\partial F}{\partial x^{i}} \frac{\partial}{\partial y^{i}}\right\} .
$$

We can consider a more general class of Legendre foliations by weakening the condition that $F$ is positive on $T_{0}^{*} M$. In this case, we obtain the Legendre foliation $\mathscr{F}_{F}$ on $F^{-1}(1)$ by a similar argument.

1.9 Symplectification. A symplectification is a canonical way of obtaining a symplectic manifold from a given contact manifold. Let $(P, H)$ be a contact manifold in the wider sense. Then the line bundle $E^{*} \subset T^{*} P$, as a $(2 n+2)$ dimensional manifold, carries the canonical symplectic 2-form $-d \tilde{\eta}$ where $\tilde{\eta}$ is the 1 -form on $E^{*}$ defined by the formula

$$
\tilde{\eta}_{v}(X)=v\left(\pi_{E *} X\right)
$$

for $v \in E^{*}$ and $X \in T_{v} E^{*}$. Moreover, for each Legendre foliation $\mathscr{F}$ of $(P, H), \pi_{E}^{*} \mathscr{F}$ is a Lagrangian foliation on $E^{*}$. Furthermore, the leaves of $\pi_{E}^{*} \mathscr{F}$ are integral submanifolds of $\tilde{\eta}$. (Here, $\pi_{E}^{*} \mathscr{F}$ denotes the foliation whose leaves are inverse images of leaves of $\mathscr{F}$.)

The proof that $-d \tilde{\eta}$ is a symplectic 2 -form is as follows. Consider a local trivialization $\eta$ of $E^{*}$ (i.e. $E_{\mid U}^{*} \simeq U \times \mathbb{R}$ defined by the correspondence $t \eta_{p} \leftrightarrow$ $(p, t)$, where $U$ is a neighborhood in $P)$. With respect to this trivialization, we have $\tilde{\eta}=t \eta$ since $\tilde{\eta}_{(p, t)}(X)=t \eta_{p}\left(\pi_{E *} X\right)=t \eta(X)$. Therefore,

$$
\begin{aligned}
(d \tilde{\eta})^{n+1} & =\{d(t \eta)\}^{n+1}=(d t \wedge \eta+t d \eta)^{n+1} \\
& =(n+1) t^{n} d t \wedge \eta \wedge(d \eta)^{n}+t^{n+1}(d \eta)^{n+1}
\end{aligned}
$$

But $(d \eta)^{n+1}=0$ since $d \eta$ has rank $n$, and $(-d \tilde{\eta})^{n+1} \neq 0$ follows from the fact that $\eta \wedge(d \eta)^{n}$ is the pull back by $\pi_{E}$ of the volume form on $P$.

In general, a $(2 n+2)$-dimensional manifold with 1-form $\tilde{\eta}$ such that $-d \tilde{\eta}$ is a symplectic 2-form is called an exact symplectic manifold.

1.10 Definition. A Lagrangian foliation of an exact symplectic manifold is a foliation of $P$ by $(n+1)$-dimensional integral submanifold of $\tilde{\eta}$.

For example, the foliation defined by the fibers of $\pi: T^{*} M \rightarrow M$ is a Lagrangian foliation of the exact symplectic manifold $\left(T^{*} M, \zeta\right)$. Given a Legendre foliation $\mathscr{F}$, the foliation $\pi_{E}^{*} \mathscr{F}$ is another example of a Lagrangian foliation of an exact symplectic manifold, $\left(E^{*}, \tilde{\eta}\right)$.

\section{NORMAL FORM FOR LEGENDRE FOLIATIONS}

In this section, we prove that every Legendre foliation $\mathscr{F}$ is locally equivalent to one of the form $\mathscr{F}_{F}$. This result is called the Normal Form Theorem. As a corollary of the Normal Form Theorem, it follows that all Legendre foliations in the wider sense are locally equivalent. The proof is based on the local equivalence of the Lagrangian foliations of exact symplectic manifolds. 
2.1 Local equivalence of Lagrangian foliations. Our theorem on the local equivalence of Lagrangian foliation of exact symplectic manifolds is similar to Weinstein's result on Lagrangian foliations of symplectic manifolds:

Proposition [W]. Let $\widetilde{F}$ be a Lagrangian foliation on a symplectic manifold $\left(\widetilde{P}^{2 n+2}, \Omega\right)$, and $p \in \widetilde{P}$. Then there is a neighborhood $U$ of $p$ and a local diffeomorphism $\phi: U \rightarrow T^{*} \mathbb{R}^{n+1}$ such that $\phi(p)=(0,0) \in \mathbb{R}^{n+1} \times \mathbb{R}^{n+1} \simeq$ $T^{*} \mathbb{R}^{n+1}$, and

(1) the pull back under $\phi$ of the canonical symplectic 2-form on $T^{*} \mathbb{R}^{n+1}$ is $\Omega$,

(2) $\phi$ sends the leaves of $\widetilde{F}$ into the fibers of $\pi: T^{*} \mathbb{R}^{n+1} \rightarrow \mathbb{R}^{n+1}$.

In particular, all Lagrangian foliations are locally equivalent. Furthermore, the leaves of a Lagrangian foliation have a natural flat affine structure.

In the case of Lagrangian foliations on an exact symplectic manifold, we prove the following theorem.

2.2 Theorem. Let $U$ be a neighborhood of a point in an exact symplectic manifold $(\widetilde{P}, \tilde{\eta})$ with Lagrangian foliation $\widetilde{\mathscr{F}}$. Without loss of generality, we may assume that the leaf space $U / \widetilde{F}$ of the restriction of $\widetilde{\mathscr{F}}$ to $U$ is a manifold. Then there is a unique local diffeomorphism

$$
\psi: U \rightarrow T^{*}(U / \widetilde{F})
$$

such that

(1) $\psi^{*} \zeta=\tilde{\eta}$ where $\zeta$ is the canonical 1 -form on $T^{*}(U / \widetilde{\mathscr{F}})$,

(2) $\pi \circ \psi=\pi_{U}$ where $\pi$ and $\pi_{U}: U \rightarrow U / \widetilde{F}$ are projection maps (i.e. each leaf of the foliation of $U$ is mapped into the fiber of $\left.T^{*}(U / \widetilde{F})\right)$.

Proof. To prove the theorem, we want to show (i) the construction of $\psi$, (ii) that the equations $\psi^{*} \zeta=\tilde{\eta}$ and $\pi_{U}=\pi \circ \psi$ hold, (iii) that $\psi$ is a local diffeomorphism, and (iv) the uniqueness of $\psi$ satisfying (1) and (2).

(i) To define the map $\psi$, consider the bundle map

$$
\Psi: \widetilde{N^{*}} \rightarrow T^{*}(U / \widetilde{F})
$$

defined as follows: Let $\widetilde{N}^{*}{ }_{\mid U} \rightarrow U$ be the conormal bundle to $\widetilde{F}$ restricted to $U$. The derivative of the projection map $\pi_{U}$ defines a bundle map $\pi_{U_{*}}$ between $\tilde{N}$ and the tangent bundle $T(U / \widetilde{\mathscr{F}})$ with $\pi_{U *}: \widetilde{N}_{p} \rightarrow T_{\pi_{U}(p)}(U / \widetilde{F})$ an isomorphism for all $p \in U$. The bundle map $\Psi$ at $p \in U$ is defined by the equation $\Psi_{p}=\left(\pi_{U}^{*}\right)^{-1}$ with $\Psi_{p}$ a vector space isomorphism. 
We define $\psi$ by the composition $\Psi \circ \tilde{\eta}$ where $\tilde{\eta}$ is regarded as a map from $U$ to $\widetilde{N}^{*}$ so that the following diagram commutes:

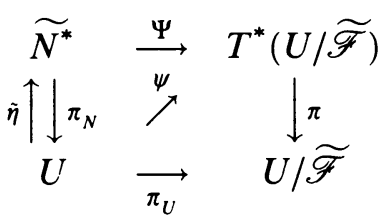

(ii) To prove the identity $\psi^{*} \zeta=\tilde{\eta}$, let $\zeta_{N}$ denote the pull back of the canonical 1-form on $T^{*} U$ to $\widetilde{N}^{*}$ where $\widetilde{N}^{*}$ is regarded as a submanifold of $T^{*} U$. We claim that $\Psi^{*} \zeta=\zeta_{N}$, and $\tilde{\eta}^{*} \zeta_{N}=\tilde{\eta}$. Then it follows that

$$
\psi^{*} \zeta=(\Psi \circ \tilde{\eta})^{*} \zeta=\tilde{\eta}^{*} \circ \Psi^{*} \zeta=\tilde{\eta}^{*} \zeta_{N}=\tilde{\eta} .
$$

To prove $\Psi^{*} \zeta=\zeta_{N}$, choose $w \in \widetilde{N^{*}}$ and $v \in T^{*}(U / \widetilde{\mathscr{F}})$ such that $\Psi(w)=v$ (equivalently $\pi_{U}^{*}(v)=w$ ). Using the commutativity of the diagram, compute as follows:

$$
\begin{aligned}
\left(\Psi^{*} \zeta\right)_{w}(X) & =\zeta_{v}\left(\Psi^{*} X\right)=v\left(\pi_{*} \circ \Psi_{*}(X)\right)=v\left(\pi_{U^{*}} \circ \pi_{N^{*}}(X)\right) \\
& =\left(\pi_{U}^{*} v\right)\left(\pi_{N^{*}}(X)\right)=w\left(\pi_{N^{*}}(X)\right)=\zeta_{N}(X)
\end{aligned}
$$

where $X \in T_{w} \widetilde{N^{*}}$.

The equation $\tilde{\eta}^{*} \zeta_{N}=\tilde{\eta}$ is a consequence of the calculation

$$
\left(\tilde{\eta}^{*} \zeta_{N}\right)(Y)=\zeta_{N}\left(\tilde{\eta}_{*} Y\right)=\tilde{\eta}_{p}\left(\pi_{N^{*}} \circ \tilde{\eta}_{*}(X)\right)=\tilde{\eta}_{p}(Y) .
$$

where $p \in U$ and $Y \in T_{p} U$.

(iii) The fact that $\psi$ is a local diffeomorphism can be seen as follows: Let $\left(x^{i}, y^{j}\right)$ be the coordinates on $T^{*} U$ induced from coordinates $\left(x^{i}\right)$ on $U$. Then $\psi^{*} d x^{i}$ and $\psi^{*} d y^{i}$ form a local coframe since $\psi^{*}\left(\sum_{i=1}^{n+1} d y^{i} \wedge d x^{i}\right)^{n+1}=$ $\psi^{*}(d \zeta)^{n+1}=(d \tilde{\eta})^{n+1} \neq 0$. By the inverse function theorem, $\psi$ is a local diffeomorphism.

(iv) For uniqueness, suppose that $\psi^{\prime}$ is another map satisfying (1) and (2). Then from the commutativity of the diagram above, $x^{i} \circ \psi=x^{i} \circ \psi^{\prime}$. Also, we have $y^{i} \circ \psi=y^{i} \circ \psi^{\prime}$ since

$$
\sum_{i=1}^{n+1}\left(y^{i} \circ \psi\right)\left(\psi^{*} d x^{i}\right)=\psi^{*} \zeta=\psi^{\prime *} \zeta=\sum_{i=1}^{n+1}\left(y^{i} \circ \psi^{\prime}\right)\left(\psi^{*} d x^{i}\right) .
$$

The following corollary is a result of Theorem 2.2.

2.3 Corollary. Suppose that $U$ is an open subset of $T^{*} M$ such that $\pi(U)=$ $M$, and $U / \mathscr{F}$ is a manifold where $\mathscr{F}$ is the foliation defined by fibers of $\pi: U \rightarrow$ $M$. If $\phi: U \rightarrow T^{*} M$ is a smooth map satisfying the properties: (i) $\phi^{*} \zeta=\zeta$ and (ii) $\pi \circ \phi=\pi$, then $\phi$ is the identity map.

Proof. Apply Theorem 2.2 to the exact symplectic manifold $(U, \zeta)$ with the Lagrangian foliation $\mathscr{F}$. Note that, since $U / \mathscr{F}=M$, we have the map 
$\psi: U \rightarrow T^{*} M$, and the maps $\phi, \psi$, and id: $U \rightarrow T^{*} M$ satisfy the properties (i) and (ii). Therefore, from uniqueness of $\psi$ in Theorem 2.2, it follows that $\phi=\psi=\mathrm{id}$.

2.4 The Normal Form Theorem. Recall that, given a Legendre foliation $\mathscr{F}$, we obtain a Lagrangian foliation $\pi_{E}^{*} \mathscr{F}$ of the symplectification. We prove the Normal Form Theorem by applying Theorem 2.2 to $\pi_{E}^{*} \mathscr{F}$ :

2.5 Normal Form Theorem. Any Legendre foliation is locally equivalent to one of the form $\mathscr{F}_{F}$. In particular, all the Legendre foliations in the wider sense are locally equivalent to the canonically defined Legendre foliation on $\mathbb{P}_{+}^{*} M$. The foliation $\mathscr{F}_{F}$ is called the normal form of $\mathscr{F}$.

Proof. Let $\mathscr{F}$ be a Legendre foliation of a contact manifold $(P, \eta)$ and $V$ be a neighborhood of a point in $P$. Again, without loss of generality, we may assume that the leaf space $V / \mathscr{F}$ of the restriction of $\mathscr{F}$ to $V$ is a manifold. If we let $U=\pi_{E}^{-1}(V) \subset E^{*}$, then $U$ is an open set such that the leaf space $U / \widetilde{F}$ is a manifold since $U / \widetilde{F}=V / \mathscr{F}$. Therefore, by applying the previous theorem to the Lagrangian foliation $\pi_{E}^{*} \mathscr{F}$, we obtain a local diffeomorphism $\psi: U \rightarrow T^{*}(V / \mathscr{F})$ satisfying the properties (1) and (2). Considering $\eta$ as a map from $V$ to $U$, let $\phi_{\eta}$ be the composition map

$$
\phi_{\eta}: V \stackrel{\eta}{\longrightarrow} U \stackrel{\psi}{\longrightarrow} T^{*}(V / \mathscr{F}) .
$$

We claim that $\phi_{\eta}$ maps $V$ diffeomorphically into $S_{F}^{*}(V / \mathscr{F})$ for some $F$, and has the properties that (i) $\phi_{\eta}^{*} \zeta=\eta$ and (ii) $\phi_{\eta}^{*} \mathscr{F}_{F}=\mathscr{F}_{\mid V}$ where $\mathscr{F}_{F}$ is the Legendre foliation on $S_{F}^{*}(V / \mathscr{F})$ and $\mathscr{F}_{\mid V}$ is the restriction of $\mathscr{F}$ to $V$. But property (i) is clear since $\phi_{\eta}^{*} \zeta=(\psi \circ \eta)^{*} \zeta=\eta^{*}\left(\psi^{*} \zeta\right)=\eta^{*} \tilde{\eta}=\eta$. Condition (ii) follows from property (2) of $\psi$ in the previous theorem and the identity $\eta^{*}\left(\pi_{E}^{*} \mathscr{F}\right)=\mathscr{F}$.

To define $F$, consider the trivialization of the line bundle $U \simeq V \times \mathbb{R}$ defined by the correspondence $t \eta_{p} \leftrightarrow(p, t)$. Define $F: \psi(U) \rightarrow \mathbb{R}$ by $F=\pi_{2} \circ \psi^{-1}$ where $\pi_{2}: U \rightarrow \mathbb{R}$ is the projection defined by $\pi_{2}(p, t)=t$. That $F$ satisfies the homogeneity condition follows from the fact that $\psi$ is a vector bundle map: For each $p \in V, \psi_{p}$ is linear since

$$
\psi(p, t)=\Psi\left(\tilde{\eta}_{(p, t)}\right)=\Psi\left(t \eta_{(p, t)}\right)=t \Psi\left(\eta_{(p, t)}\right)=t \Psi\left(\tilde{\eta}_{(p, 1)}\right)=t \psi(p, 1) .
$$

Therefore, $F$ defines the Legendre foliation $\mathscr{F}_{F}$ on $S_{F}^{*}(V / \mathscr{F})$ with contact 1-form $\eta_{F}$ defined by the pull back of $\zeta$ to $S_{F}^{*}(V / \mathscr{F})$, and $\phi_{\eta}$ is a diffeomorphism from $V$ onto $S_{F}^{*}(V / \mathscr{F})$.

2.6 Corollary. Let $\mathscr{F}_{F_{s}}$ be Legendre foliations on contact manifolds $\left(S_{F_{s}}^{*} M_{s}, \eta_{s}\right)$ for $s=1,2$ with positive $F_{s}$. If $\phi: S_{F_{1}}^{*} M_{1} \rightarrow S_{F_{2}}^{*} M_{2}$ is a diffeomorphism such that (i) $\phi^{*} \eta_{2}=\eta_{1}$ and (ii) $\phi^{*} \mathscr{F}_{F_{2}}=\mathscr{F}_{F_{1}}$, then there is a diffeomorphism $f: M_{1} \rightarrow M_{2}$ such that $F_{1} \circ f^{*}=F_{2}$ and $\phi=\left(f^{*}\right)_{\mid S_{F_{1}}^{*} M_{1}}^{-1}$. 
Proof. From the assumption (ii), there is a diffeomorphism $f$ such that the following diagram commutes:

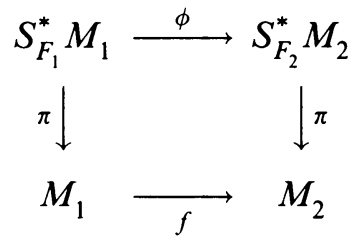

We first prove the identity $\phi=\left(f^{*}\right)_{\mid S_{F_{1}}^{*} M_{1}}^{-1}$. Then, the identity $F_{1} \circ f=F_{2}$ follows since $\left(f^{*}\right)^{-1}$ maps $S_{F_{1}}^{*} M_{1}$ diffeomorphically onto $S_{F_{2}}^{*} M_{2}$ and $F$ is homogeneous function of degree 1 (condition (1.6)).

To show that $\phi=\left(f^{*}\right)_{\mid S_{F_{1}}^{*} M_{1}}^{-1}$, it suffices to prove that $\phi \circ f^{*}=$ id. By Corollary 2.3 applied to the map $\phi \circ f^{*}$ we must only show that $\phi \circ f^{*}$ satisfies two conditions (i): $\left(\phi \circ f^{*}\right)^{*} \zeta=\zeta$ and (ii): $\pi \circ\left(\phi \circ f^{*}\right)=\pi$. Note that the condition $\pi \circ\left(\phi \circ f^{*}\right)=\pi$ is clearly satisfied.

To show $\left(\phi \circ f^{*}\right)^{*} \zeta=\zeta$, recall that $T_{0}^{*} M$ denotes the set of nonzero covectors, and consider the extension of $\phi$ to the diffeomorphism from $T_{0}^{*} M_{1}$ to $T_{0}^{*} M_{2}$ defined by $\phi(t v)=t \phi(v)$ for $t>0$ and $v \in S_{F_{1}}^{*} M_{1}$. Also, let $\rho: T^{*} M_{s} \rightarrow S_{F_{s}}^{*} M_{s}$ denotes the radial projection. Clearly then, $\rho \circ \phi=\phi \circ \rho$.

We claim that $\phi^{*} \zeta=\zeta$ and $\left(f^{*}\right)^{*} \zeta=\zeta$. The identity $\left(\phi \circ f^{*}\right)^{*} \zeta=\zeta$ follows. Note that the canonical 1-form $\zeta$ on $T^{*} M_{s}$ can be expressed by $\zeta_{t v}=t\left(\rho^{*} \eta_{s}\right)_{t v}$ on $T^{*} M_{s}$. (This can be easily seen from the facts that $\eta_{s}$ is the restriction of $\zeta$ to $S_{F_{s}}^{*} M_{s}$ and that $\zeta$ is homogenious of degree 1.) Using this, we prove $\phi^{*} \zeta=\zeta^{s}$ :

$$
\left(\phi^{*} \zeta\right)_{t v}=t\left(\phi^{*} \circ \rho^{*} \eta_{2}\right)_{t v}=t\left(\rho^{*} \circ \phi^{*} \eta_{2}\right)_{t v}=t\left(\rho^{*} \eta_{1}\right)_{t v}=\zeta_{t v} .
$$

Also, from the definition (1.5) of $\zeta$, we can easily check the second identity $\left(f^{*}\right)^{*} \zeta=\zeta$ :

$$
\begin{aligned}
\left\{\left(f^{*}\right)^{*} \zeta\right\}_{v}(X) & =\zeta\left\{\left(f^{*}\right)_{*} X\right\}=f^{*}(v)\left\{\pi_{*} \circ\left(f^{*}\right)_{*} X\right\} \\
& =f^{*}(v)\left(f_{*} \circ \pi_{*} X\right)=v\left(\pi_{*} X\right)=\zeta_{v}(X) .
\end{aligned}
$$

for $v \in T^{*} M_{2}$ and $X \in T_{v}\left(T^{*} M_{2}\right)$.

\section{LOCAL INVARIANTS}

3.1 The fundamental quadratic form $\Pi$ and the invariant $G$. To determine when two Legendre foliations are locally equivalent, we introduce the local invariants, $\Pi \in \Gamma\left(\odot^{2} L^{*}\right)$, the fundamental quadratic form, and $\mathbf{G} \in \Gamma\left(\odot^{3} L^{*}\right)$.

In the case of Legendre foliations of the form $\mathscr{F}_{F}$, the invariants $\Pi$ and $\mathbf{G}$ are given by the restriction to $L$ of the symmetric forms

$$
\boldsymbol{\Pi}=F_{i j} d y^{i} \otimes d y^{j} \quad \text { and } \quad \mathbf{G}=\frac{1}{2} F_{i j k} d y^{i} \otimes d y^{j} \otimes d y^{k} .
$$


(Recall that $F_{i}$ and $F_{i j}$ denote the derivatives of $F$ with respect to $y^{i}$ and $y^{j}$.) Given an arbitrary Legendre foliation $\mathscr{F}$, the normal form theorem provides a canonical identification of $\mathscr{F}$ with $\mathscr{F}$, and the invariants $\Pi$ and $\mathbf{G}$ can be also defined by the formulas (3.2). However, the quantities $\Pi$ and $\mathbf{G}$ can be invariantly defined in terms of Lie derivatives:

3.3 Proposition. Let $X, Y, Z$ be tangential vector fields to $\mathscr{F}$.

$$
\begin{aligned}
& \Pi(X, Y)=-\left\{\mathscr{L}_{X} \mathscr{L}_{Y} \eta\right\}\left(\mathbf{X}_{\eta}\right) \\
& \mathbf{G}(X, Y, Z)=\frac{1}{2}\{X(\boldsymbol{\Pi}(Y, Z))+Y(\boldsymbol{\Pi}(X, Z))+Z(\boldsymbol{\Pi}(X, Y)) \\
&\left.+\left(\mathscr{L}_{Y} \mathscr{L}_{X} \mathscr{L}_{Z} \eta+\mathscr{L}_{Z} \mathscr{L}_{X} \mathscr{L}_{Y} \eta\right)\left(\mathbf{X}_{\eta}\right)\right\} .
\end{aligned}
$$

Proof. We prove these equations when $X=X_{\alpha}, Y=X_{\beta}, Z=X_{\gamma}$ for a local frame $\left(X_{\rho}\right)$ of $L$ on a neighborhood $U \subset S_{F}^{*} M$. By the Normal Form Theorem, we may assume that $X_{\alpha}=u_{\alpha}^{i} \frac{\partial}{\partial y^{i}}$ where $u_{\alpha}^{i}$ are smooth functions defined on $T^{*} M$ satisfying $d F\left(X_{\alpha}\right)=F_{i} u_{\alpha}^{i}=0$.

(i) The identity (3.4) follows from the following computation: Note that $\mathscr{L}_{X_{\alpha}} d x^{i}=d\left(X_{\alpha} x^{i}\right)=0$ and $d x^{i}\left(\mathbf{X}_{\eta}\right)=F_{i}$ from $(1.8)$.

$$
\begin{aligned}
\left\{\mathscr{L}_{X_{\alpha}} \mathscr{L}_{X_{\beta}} \eta\right\}\left(\mathbf{X}_{\eta}\right) & =\left\{\mathscr{L}_{X_{\alpha}} \mathscr{L}_{X_{\beta}}\left(\sum_{i=1}^{n+1} y^{i} d x^{i}\right)\right\}\left(\mathbf{X}_{\eta}\right) \\
& =\sum_{i=1}^{n+1}\left\{\mathscr{L}_{X_{\alpha}}\left(u_{\beta}^{i} d x^{i}\right)\right\}\left(\mathbf{X}_{\eta}\right) \\
& =\sum_{i=1}^{n+1}\left\{\left(X_{\alpha} u_{\beta}^{i}\right) d x^{i}\right\}\left(\mathbf{X}_{\eta}\right)=\sum_{i=1}^{n+1}\left\{\left(X_{\alpha} u_{\beta}^{i}\right) F_{i}\right\} \\
& =-\sum_{i=1}^{n+1}\left\{u_{\beta}^{i}\left(X_{\alpha} F_{i}\right)\right\}=-F_{i j} u_{\alpha}^{j} u_{\beta}^{i} \\
& =-\Pi\left(X_{\alpha}, X_{\beta}\right) .
\end{aligned}
$$

(ii) To prove the identity (3.5), recall from (i) that we have the identity $\mathscr{L}_{X_{\alpha}} \mathscr{L}_{X_{\beta}} \eta=\sum_{i=1}^{n+1}\left(X_{\alpha} u_{\beta}^{i}\right) d x^{i}$. Using this, compute

$$
\begin{aligned}
\left\{\mathscr{L}_{X_{i}} \mathscr{L}_{X_{\beta}} \mathscr{L}_{X_{\eta}} \eta\right\}\left(\mathbf{X}_{\eta}\right) & =\left\{\mathscr{L}_{X_{i}}\left(\sum_{i=1}^{n+1}\left(X_{\beta} u_{\gamma}^{i}\right) d x^{i}\right)\right\}\left(\mathbf{X}_{\eta}\right) \\
& =\sum_{i=1}^{n+1}\left\{\left(X_{\alpha} X_{\beta} u_{\eta}^{i}\right) d x^{i}\right\}\left(\mathbf{X}_{\eta}\right)=\left(X_{\alpha} X_{\beta} u_{\gamma}^{i}\right) F_{i}
\end{aligned}
$$




$$
\begin{aligned}
& =X_{\alpha}\left\{\left(X_{\beta} u_{\gamma}^{i}\right) F_{i}\right\}-\left(X_{\beta} u_{\gamma}^{i}\right)\left(X_{\alpha} F_{i}\right) \\
& =-X_{\alpha}\left\{u_{\gamma}^{i}\left(X_{\beta} F_{i}\right)\right\}-\left(X_{\beta} u_{\gamma}^{i}\right)\left(X_{\alpha} F_{i}\right) \\
& =-X_{\alpha}\left\{u_{\gamma}^{i} u_{\beta}^{j} F_{i j}\right\}-\left(X_{\beta} u_{\gamma}^{i}\right) u_{\alpha}^{j} F_{i j} \\
& =-X_{\alpha}\left\{\Pi\left(X_{\beta}, X_{\gamma}\right)\right\}-\left(X_{\beta} u_{\gamma}^{i}\right) u_{\alpha}^{j} F_{i j} .
\end{aligned}
$$

From this, we can directly calculate the right-hand side of (3.5)

$$
\begin{aligned}
& \frac{1}{2}\left\{X_{\alpha}\left(F_{i j} u_{\beta}^{i} u_{\gamma}^{j}\right)-\left(X_{\alpha} u_{\beta}^{i}\right) u_{\gamma}^{j} F_{i j}-\left(X_{\alpha} u_{\gamma}^{i}\right) u_{\gamma}^{j} F_{i j}\right\} \\
& \quad=\frac{1}{2}\left(X_{\alpha} F_{i j}\right) u_{\beta}^{i} u_{\gamma}^{j}=\frac{1}{2} F_{i j k} u_{\beta}^{i} u_{\gamma}^{j} u_{\alpha}^{k}=\mathbf{G}\left(X_{\alpha}, X_{\beta}, X_{\gamma}\right) .
\end{aligned}
$$

3.6 The Bott partial connection and fundamental invariants. The fundamental invariants of Legendre foliations can be often more conveniently described in terms of the Bott partial connection and moving frames. For instance, the invariant $\mathbf{G}$ has a relatively simple expression in terms of the Bott partial connection. (See Proposition 3.19 below.)

Before defining the Bott partial connection, we briefly introduce relative forms, and the relative exterior derivative. For more details of these, see [BGJ, $\mathrm{KT}$, and DK]. A relative $k$-form is a section of $\Lambda^{k} L^{*}$. Let $\boldsymbol{\Omega}_{\mathscr{F}}^{k}(P)$ denote the set of all relative $k$-forms. The relative exterior derivative $d_{\mathscr{F}}: \boldsymbol{\Omega}_{\mathscr{F}}^{k}(P) \rightarrow$ $\boldsymbol{\Omega}_{\mathscr{F}}^{k+1}(P)$ is defined as follows. Note that the vector bundle obtained by restricting $L^{*}$ to a leaf $\Sigma \in \mathscr{F}$ is simply the cotangent bundle $T^{*} \Sigma$ of $\Sigma$. If $v$ is a relative form, we define the relative exterior derivative $d_{\mathscr{F}} v$ of $v$ to be the ordinary exterior differential of $v$ restricted on each leaf $\Sigma$. More precisely, if $\imath^{*}: \boldsymbol{\Omega}^{k}(P) \rightarrow \boldsymbol{\Omega}_{\mathscr{F}}^{k}(P)$ denotes the pull back of a $k$-form on $P$ to a relative $k$-form, the relative exterior derivative $d_{\mathscr{F}}$ is defined by the following commutative diagram:

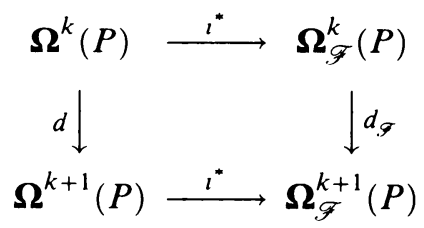

Definition 3.7. For an arbitrary vector bundle $B \rightarrow P$, a partial connection $\nabla^{B}$ on $B$ is an $\mathbb{R}$-linear map

$$
\nabla^{B}: \Gamma(B) \longrightarrow \Gamma\left(L^{*} \otimes B\right)
$$

satisfying the equation $\nabla^{B}(f v)=f \nabla^{B} v+d_{\mathscr{F}} f \otimes v$, for $f \in C^{\infty}(M)$, and $v \in \Gamma(B)$. The curvature $R$ is a $\operatorname{Hom}(B, B)$-valued relative 2-form defined by $R(X, Y) v=\left[\nabla_{X}^{B}, \nabla_{Y}^{B}\right] v-\nabla_{[X, Y]}^{B} v$, where $X, Y$ are tangential vector fields and $[$, ] denotes the commutator.

If $v=\left(v^{\alpha}\right)$ is a local frame for $B$, there is a matrix valued 1 -form $\omega$ called relative partial connection form, and a relative 2 -form $\Omega$ called the curvature 
matrix, determined by the conditions

$$
\nabla^{B} v=-\omega \otimes v, \quad R v=-\Omega \otimes v .
$$

From this the equation $\Omega=d_{\mathscr{F}} \omega+\omega \wedge \omega$ follows.

3.10 Definition. The Bott partial connection on $N^{*}$ is a partial connection defined by

$$
\nabla_{X}^{N} v=\mathscr{L}_{X} v=i(X) d v
$$

where $X$ and $v$ are tangential vector field and a section of $N^{*}$.

It can be easily checked that $\nabla^{N}$ is a partial connection. Moreover, the Bott partial connection is flat since $R(X, Y)=\left[\mathscr{L}_{X}, \mathscr{L}_{Y}\right] v-\mathscr{L}_{[X, Y]} v=0$.

The Bott partial connection induces a partial connection $\nabla^{Q}$ on the bundle $Q^{*}$ defined by $\nabla_{X}^{Q} v=\pi\left(\nabla_{X}^{N} v\right)$ for $X$ and $v$ are tangential vector field and a section of $Q^{*}$ where $\pi: N^{*} \rightarrow Q^{*}$ denotes the projection onto $Q^{*}$ (recall $N^{*}=E^{*} \oplus Q^{*}$ from (1.3)). We call $\nabla^{Q}$ the induced partial connection.

The partial connection $\nabla^{Q}$ has the following relation with the invariants $\Pi$ and $\mathbf{G}$. Let $\left\{\theta^{\alpha}\right\}$ be a local frame for $Q^{*}$. Then $\left\{\theta^{\alpha}, \eta\right\}$ is a local frame for $N^{*}$, and the relative partial connection form $\omega=\left(\omega_{j}^{i}\right)$ of $\nabla^{N}$ is determined by the equation:

$$
\nabla^{N}\left(\begin{array}{c}
\theta^{\alpha} \\
\eta
\end{array}\right)=-\left(\begin{array}{cc}
\omega_{\beta}^{\alpha} & \omega_{n+1}^{\alpha} \\
\omega_{\beta}^{n+1} & 0
\end{array}\right) \otimes\left(\begin{array}{c}
\theta^{\beta} \\
\eta
\end{array}\right)
$$

Note that $\omega_{n+1}^{n+1}=0$ since $\nabla_{X}^{N} \eta=\Phi(X) \in \Gamma\left(Q^{*}\right)$, for $X \in \Gamma(L)$ is a section of $Q^{*}$. Clearly, $\left(\omega_{\beta}^{\alpha}\right)$ is the relative connection form for $\nabla^{Q}$. Since the curvature of the Bott connection vanishes, the relative connection form $\left(\omega_{j}^{i}\right)$ satisfies the equation

$$
d_{\mathscr{F}}\left(\begin{array}{cc}
\omega_{\beta}^{\alpha} & \omega_{n+1}^{\alpha} \\
\omega_{\beta}^{n+1} & 0
\end{array}\right)+\left(\begin{array}{cc}
\omega_{\gamma}^{\alpha} & \omega_{n+1}^{\alpha} \\
\omega_{\gamma}^{n+1} & 0
\end{array}\right) \wedge\left(\begin{array}{cc}
\omega_{\beta}^{\gamma} & \omega_{n+1}^{\gamma} \\
\omega_{\beta}^{n+1} & 0
\end{array}\right)=0 .
$$

Expansion of equation (3.13) yields the following identities:

$$
\begin{aligned}
& d_{\mathscr{F}} \omega_{\beta}^{\alpha}+\omega_{\gamma}^{\alpha} \wedge \omega_{\beta}^{\gamma}+\omega_{n+1}^{\alpha} \wedge \omega_{\beta}^{n+1}=0, \\
& d_{\mathscr{F}} \omega_{n+1}^{\alpha}+\omega_{\gamma}^{\alpha} \wedge \omega_{n+1}^{\gamma}=0, \\
& d_{\mathscr{F}} \omega_{\beta}^{n+1}+\omega_{\gamma}^{n+1} \wedge \omega_{\beta}^{\gamma}=0, \\
& \omega_{\gamma}^{n+1} \wedge \omega_{n+1}^{\gamma}=0 .
\end{aligned}
$$

3.18 Lemma. The relative 1-forms $\omega_{\alpha}^{n+1}$ constitute a local frame for $L^{*}$.

Proof. Let $X_{\alpha}$ be the local frame for $L$ determined by the equation $\Phi\left(X_{\alpha}\right)=$ $-\theta^{\alpha}$. The independence of $\omega_{\alpha}^{n+1}$ follows from the fact that $\omega_{\alpha}^{n+1}$ is a dual coframe of $X_{\alpha}$ :

$$
\theta^{\alpha}=-\Phi\left(X_{\alpha}\right)=-i\left(X_{\alpha}\right) d \eta=-\nabla_{X_{\alpha}} \eta=\omega_{\beta}^{n+1}\left(X_{\alpha}\right) \theta^{\beta} .
$$

The invariants $\Pi$ and $\mathbf{G}$ can be expressed as follows. 
3.19 Proposition. There is a symmetric matrix valued function $\left(c^{\alpha \beta}\right)$ such that the identities

$$
\begin{aligned}
& \boldsymbol{\Pi}=-c^{\alpha \beta} \omega_{\alpha}^{n+1} \otimes \omega_{\beta}^{n+1}, \\
& \mathbf{G}=\frac{1}{2}\left\{d_{\mathscr{F}} c^{\alpha \beta}+c^{\alpha \gamma} \omega_{\gamma}^{\beta}+c^{\beta \gamma} \omega_{\gamma}^{\alpha}\right\} \otimes \omega_{\alpha}^{n+1} \otimes \omega_{\beta}^{n+1}
\end{aligned}
$$

are satisfied.

Proof. From equation (3.17), and since $\omega_{\alpha}^{n+1}$ are linearly independent, it follows that there is a symmetric matrix valued function $c^{\alpha \beta}$ such that $\omega_{n+1}^{\alpha}=$ $c^{\alpha \beta} \omega_{\beta}^{n+1}$.

(i) Equation (3.20) is a consequence of the calculation

$$
\begin{aligned}
\Pi\left(X_{\alpha}, X_{\beta}\right) & =-\left\{\mathscr{L}_{X_{\alpha}} \mathscr{L}_{X_{\beta}} \eta\right\}\left(\mathbf{X}_{\eta}\right)=-\left\{\nabla_{X_{\alpha}}^{N}\left(-\omega_{\sigma}^{n+1}\left(X_{\beta}\right) \theta^{\sigma}\right)\right\}\left(\mathbf{X}_{\eta}\right) \\
& =\left\{\nabla_{X_{\alpha}}^{N} \theta^{\beta}\right\}\left(\mathbf{X}_{\eta}\right)=-c^{\alpha \beta} .
\end{aligned}
$$

(ii) We prove the equation (3.21) by computing $\mathbf{G}\left(X_{\alpha}, X_{\beta}, X_{\gamma}\right)$ using the invariant definition of $\mathbf{G}$ given by the formula (3.5). To do this, we first compute

$$
\begin{aligned}
\left\{\mathscr{L}_{X_{\beta}} \mathscr{L}_{X_{\alpha}} \mathscr{L}_{X_{\gamma}} \eta\right\}\left(\mathbf{X}_{\eta}\right)=\left\{\nabla_{X_{\beta}}^{N} \nabla_{X_{\alpha}}^{N} \nabla_{X_{\gamma}}^{N} \eta\right\}\left(\mathbf{X}_{\eta}\right)=-\left\{\nabla_{X_{\beta}}^{B} \nabla_{X_{\alpha}}^{B} \theta^{\gamma}\right\}\left(\mathbf{X}_{\eta}\right) \\
=\left\{\nabla_{X_{\beta}}^{N}\left(\omega_{\sigma}^{\gamma}\left(X_{\alpha}\right) \theta^{\sigma}+\omega_{n+1}^{\gamma}\left(X_{\alpha}\right) \eta\right)\right\}\left(\mathbf{X}_{\eta}\right) \\
=-\omega_{\sigma}^{\gamma}\left(X_{\alpha}\right) \omega_{n+1}^{\sigma}\left(X_{\beta}\right)+\left\{\nabla_{X_{\beta}}^{N}\left(c^{\gamma \alpha} \eta\right)\right\}\left(\mathbf{X}_{\eta}\right) \\
=-c^{\beta \sigma} \omega_{\sigma}^{\gamma}\left(X_{\alpha}\right)+X_{\beta} c^{\gamma \alpha} .
\end{aligned}
$$

Using this and the identity $X_{\alpha}\left(\Pi\left(X_{\beta}, X_{\gamma}\right)\right)=X_{\alpha} c^{\beta \gamma}$ yields the formula

$$
\mathbf{G}\left(X_{\alpha}, X_{\beta}, X_{\gamma}\right)=\frac{1}{2}\left\{X_{\alpha} c^{\beta \gamma}+c^{\gamma \sigma} \omega_{\sigma}^{\beta}\left(X_{\alpha}\right)+c^{\beta \sigma} \omega_{\sigma}^{\gamma}\left(X_{\alpha}\right)\right\} .
$$

3.22 The canonical affine connection on leaves. Recall that the leaves of a Lagrangian foliation have a natural flat affine structure [W]. In the case of Legendre foliations, there is a natural affine connection on leaves, which is flat precisely when $\Pi=0$.

To define it, proceed as follows. Let $\nabla^{L}$ denote the partial connection on $L$ obtained from the induced connection by identifying $L$ with $Q^{*}$ by the vector bundle isomorphism $\Phi: L \rightarrow Q^{*}$. More precisely, $\nabla^{L}$ is defined by the formula

$$
\nabla_{X}^{L} Y=\Phi^{-1} \circ \nabla_{X}^{Q} \circ \Phi(Y) \text {. }
$$

The partial connection $\nabla^{L}$ induces an ordinary affine connection on the leaf $\Sigma$. Let $X$ and $Y$ be vector fields on $\Sigma$ supported on a appropriately small neighborhood of a point in $\Sigma$. Without loss of generality, we may assume that $X$ and $Y$ extend to tangential vector fields $\tilde{X}$ and $\tilde{Y}$ on $P$. Recall that the restriction of the bundle $L^{*}$ to $\Sigma$ is $T^{*} \Sigma$. Similarly, we have $L_{\mid \Sigma}=T \Sigma$. The 
affine connection on $\Sigma$ is defined by the restriction to $\Sigma$ of $\nabla_{\widetilde{X}}^{L} \widetilde{Y}$. It is not difficult to check that it is well-defined and independent of the choice of the extensions $\widetilde{X}$ and $\widetilde{Y}$.

3.23 Proposition. The partial connection $\nabla^{L}$ is torsion free and has curvature

$$
R^{L}(X, Y) Z=\Pi(X, Z) Y-\Pi(Y, Z) X
$$

for tangential vector fields $X, Y$, and $Z$ on $P$. In particular, if $\Pi=0$ then $R^{L}=0$, and the canonical connection on each leaf is torsion free and flat.

Proof. To prove that $\nabla^{L}$ is torsion free, we need to show $\nabla_{X}^{L} Y-\nabla_{Y}^{L} X-$ $[X, Y]=0$. Recall that $\nabla_{X}^{Q} v=\pi\left(\nabla_{X}^{N} v\right)$ where $\pi: E^{*} \oplus Q^{*} \rightarrow Q^{*}$ is the projection.

$$
\begin{aligned}
\nabla_{X}^{L} Y & =\Phi^{-1}\left\{\nabla_{X}^{Q} \Phi(Y)\right\}=\Phi^{-1}\left\{\nabla_{X}^{Q} \nabla_{Y}^{N} \eta\right\}=\Phi^{-1}\left\{\pi\left(\nabla_{X}^{N} \nabla_{Y}^{N} \eta\right)\right\} \\
& =\Phi^{-1}\left\{\pi\left(\nabla_{Y}^{N} \nabla_{X}^{N} \eta+\nabla_{[X, Y]}^{N} \eta\right)\right\} \\
& =\Phi^{-1}\left\{\nabla_{Y}^{Q} \Phi(X)\right\}+\Phi^{-1}\{\Phi([X, Y])\}=\nabla_{Y}^{L} X+[X, Y] .
\end{aligned}
$$

To prove the identity (3.24), it suffices to check it in the special case $X=$ $X_{\alpha}, Y=X_{\beta}$, and $Z=X_{\gamma}$, where $\left(X_{\sigma}\right)$ is the local frame for $L$ such that $\Phi\left(X_{\sigma}\right)=-\theta^{\sigma}$. (See the proof of Lemma 3.18.)

$$
\begin{aligned}
R^{L}\left(X_{\alpha}, X_{\beta}\right) X_{\gamma} & =\left[\nabla_{X_{\alpha}}^{L}, \nabla_{X_{\beta}}^{L}\right] X_{\gamma}-\nabla_{\left[X_{\alpha}, X_{\beta}\right]}^{L} X_{\gamma} \\
& =-\Phi^{-1}\left\{\left[\nabla_{X_{\alpha}}^{Q}, \nabla_{X_{\beta}}^{Q}\right] \theta^{\gamma}-\nabla_{\left[X_{\alpha}, X_{\beta}\right]}^{Q} \theta^{\gamma}\right\} \\
& =-\sum_{\sigma=1}^{n}\left\{d_{\mathscr{F}} \omega_{\sigma}^{\gamma}+\omega_{\rho}^{\gamma} \wedge \omega_{\sigma}^{\rho}\right\}\left(X_{\alpha}, X_{\beta}\right) X_{\sigma} .
\end{aligned}
$$

But, from the equation (3.14), $\left\{d_{\mathscr{F}} \omega_{\sigma}^{\gamma}+\omega_{\rho}^{\gamma} \wedge \omega_{\sigma}^{\rho}\right\}\left(X_{\alpha}, X_{\beta}\right)=c^{\gamma \beta} \delta_{\sigma}^{\alpha}-c^{\gamma \alpha} \delta_{\sigma}^{\beta}$. Therefore,

$$
R^{L}\left(X_{\alpha}, X_{\beta}\right) X_{\gamma}=c^{\gamma \alpha} X_{\beta}-c^{\gamma \beta} X_{\alpha}=\Pi\left(X_{\alpha}, X_{\gamma}\right) X_{\beta}-\Pi\left(X_{\beta}, X_{\gamma}\right) X_{\alpha} .
$$

\section{Flat Legendre foliations}

The simplest class of Legendre foliations is characterized by the vanishing of $\Pi$. Such Legendre foliations are called flat, and a result analogous to the Weinstein's [W] on Lagrangian foliations holds:

4.1 Theorem. Let $\mathscr{F}$ be a flat Legendre foliation on $(P, \eta)$, and $p \in P$. Then there are coordinates $\left(x^{i}, y^{j}\right)$ around $p$ such that

$$
\eta=d x^{n+1}+\sum_{\alpha=1}^{n} y^{\alpha} d x^{\alpha},
$$


and the foliation is locally defined by $x^{i} \equiv$ constant. Consequently, all flat Legendre foliations are locally equivalent. Moreover, leaves of $\mathscr{F}$ have a natural flat affine structure.

Proof. Note that, if $\Pi=0$, then the canonical affine connection on leaves is torsion free and flat by Proposition 3.23, and hence, the leaves of $\mathscr{F}$ have a natural flat affine structure.

To show the existence of the coordinates satisfying the identity (4.2), use the normal form theorem to identify $\mathscr{F}$ locally with its normal form $\mathscr{F}_{F}$ defined on $S_{F}^{*} M \subset T^{*} M$. Then, $\Pi$ can be expressed as the restriction to $L$ of $F_{i j} d \bar{y}^{i} \otimes d \bar{y}^{j}$ (see (3.2)) where $\left(\bar{x}^{i}, \bar{y}^{j}\right)$ are coordinates on $T^{*} M$ induced from coordinates $\left(\bar{x}^{i}\right)$ on $M$.

First, we claim that $F_{i j}=0$ on $T^{*} M$ and, consequently, $F=s_{i}(\bar{x}) \bar{y}^{i}$ for some functions $s_{i}(\bar{x})$ depending only upon $\bar{x}^{i}$. This can be seen from the condition $\Pi=0$ and the fact that $F$ is of homogeneous degree 1 . For observe that, the condition $\Pi=0$ implies

$$
\left\{F_{i j} d \bar{y}^{i} \otimes d \bar{y}^{j}\right\}(X, Y)=0
$$

for tangential vector fields $X, Y$ (i.e. $d F(X)=d F(Y)=d \bar{x}^{i}(X)=d \bar{x}^{i}(Y)=$ $0)$. Note that from the homogeniety condition on $F$, we have $F_{i} \bar{y}^{i}=F$, and by differentiating this, we obtain $F_{i j} \bar{y}^{i}=0$. We now want to prove equation (4.3) for arbitrary $X, Y$. It suffices to show this for the special case when $X=\bar{y}^{i} \frac{\partial}{\partial \bar{y}^{i}}$ since $d F\left(\bar{y}^{i} \frac{\partial}{\partial \bar{y}^{i}}\right)=F_{i} \bar{y}^{i}=F=1 \neq 0$ on $S_{F}^{*} M$ :

$$
\left\{F_{i j} d \bar{y}^{i} \otimes d \bar{y}^{j}\right\}\left(\bar{y}^{k} \frac{\partial}{\partial \bar{y}^{k}}, Y\right)=F_{i j} \bar{y}^{i}\left(Y \bar{y}^{j}\right)=0
$$

for an arbitrary vector field $Y$.

To show the existence of coordinates satisfying (4.2), recall that we have $F=s_{i} \bar{y}^{i}$ for some smooth functions $s_{i}$ depending only on $\bar{x}^{i}$. Consider a vector field on $\mathbb{R}^{n+1}$ defined by $s_{i} \frac{\partial}{\partial x^{i}}$. Since the vector field $s_{i} \frac{\partial}{\partial x^{i}}$ defines a 1-dimensional foliation, we can find coordinate functions $x^{i}$ on $\mathbb{R}^{n+1}$ such that $s_{i} \frac{\partial}{\partial x^{i}}=\frac{\partial}{\partial x^{n+1}}$, and $d x^{\alpha}\left(s_{i} \frac{\partial}{\partial x^{i}}\right)=0$ for $\alpha=1, \ldots, n$. Clearly then $s_{i} \frac{\partial x^{j}}{\partial x^{i}}=\delta_{n+1}^{j}$. We claim that the coordinates $\left(x^{i}, y^{j}\right)$ on $T^{*} \mathbb{R}^{n+1}$ induced by $\left(x^{i}\right)$ satisfiy (4.2). To prove this, it suffices to show that $F=y^{n+1}$ since $\eta$ is a restriction of $\zeta=\sum_{i=1}^{n+1} y^{i} d x^{i}$. Note that $y^{i}$ and $\bar{y}^{i}$ are related by the relation $\sum_{i=1}^{n+1} y^{i} \frac{\partial x^{i}}{\partial x^{j}}=$ $\bar{y}^{j}$. Thus, if we rewrite $\mathrm{F}$ in terms of $\left(x^{i}, y^{i}\right), F=s_{i} \bar{y}^{i}=\sum_{i, j=1}^{n+1} s_{i} y^{j} \frac{\partial x^{j}}{\partial \bar{x}^{i}}=$ $\sum_{j=1}^{n+1} y^{j} \delta_{n+1}^{j}=y^{n+1}$.

\section{NONDEGENERATE LEGENDRE FOLIATIONS}

Legendre foliations with nondegenerate $\Pi$ are called nondegenerate. For such foliations, we apply Cartan's method of equivalence to obtain a set of 
local invariants. We begin by showing that there is a canonical reduction of the structure group of $P$ to the orthogonal group $O(p, q)$ where $(p, q)$ is the signature of $\Pi$. Then, we show that there is a canonical connection on $P$ characterized by certain properties. Its torsion and curvature form a set of local invariants for the foliation.

The study of nondegenerate Legendre foliations is a generalization of Chern's work on Finsler spaces [C]. In fact, we show that a Legendre foliation is locally equivalent to one of the form $\mathscr{F}_{F}$ with $F$ a Finsler metric precisely when $\Pi$ is positive definite. In [C], it is proved that $F$ is a norm induced by a Riemannian metric if and only if $\mathbf{G}$ vanishes. As a result of the normal form theorem, we show that every Legendre foliation $\mathscr{F}$ with positive definite $\Pi$ and vanishing $\mathbf{G}$ is locally equivalent to one of the form $\mathscr{F}_{F}$ with $F$ the norm of a Riemannian metric. In this case, $\mathscr{F}$ is called a Riemannian (pseudo-Riemannian if $\Pi$ is nondegenerate) Legendre foliation.

We conclude this section by showing that there is a natural almost CRstructure on $P$ resulting from the reduction of the structure group.

5.1 Reduction of structure group. To describe the canonical group reduction, introduce a symmetric 2 -form $\widetilde{\boldsymbol{\Pi}}$ on $\mathrm{P}$. To do this, define a tensor field $\lambda \epsilon$ $\Gamma\left(T^{*} P \otimes L\right)$ as follows. Let $\Phi^{*}: T P \rightarrow L^{*}$ be the vector bundle map defined by the equation $\Phi^{*}(X)=\{i(X) d \eta\}_{\mid L}$ for a vector field $X$ on $P$. From the nondegeneracy of $\Pi$, we obtain a natural isomorphism $h: L \rightarrow L^{*}$ defined by the relation $\{h(Y)\}(Z)=\Pi(Y, Z)$ for $Y, Z$ tangent to $\mathscr{F}$. The canonical tensor field $\lambda$ is then defined by the composition of bundle maps

$$
\lambda: T P \stackrel{\Phi^{*}}{\longrightarrow} L^{*} \stackrel{h^{-1}}{\longrightarrow} L \subseteq T P
$$

From this, we obtain the relation

$$
\Pi(\lambda(X), Y)=d \eta(X, Y)
$$

for $Y$ tangent to $\mathscr{F}$. The symmetric 2 -form $\widetilde{\mathbf{\Pi}}$ is defined by the formula $\widetilde{\boldsymbol{\Pi}}=\lambda^{*} \Pi$.

5.4 Theorem. There exist a canonical set of coframes, $\left(\theta^{\alpha}, \eta, \xi^{\alpha}\right)$ on $P$ determined by the conditions

(1) $\theta^{\alpha} \in \Gamma\left(Q^{*}\right)$,

(2) $d \eta=\delta_{\alpha \beta} \theta^{\alpha} \wedge \xi^{\beta}$,

(3) $\widetilde{\boldsymbol{\Pi}}=\varepsilon_{\alpha \beta} \theta^{\alpha} \otimes \theta^{\beta}$,

(4) $\mathscr{L}_{\mathbf{x}_{\eta}} \widetilde{\boldsymbol{\Pi}}=-\delta_{\alpha \beta} \theta^{\alpha} \otimes \xi^{\beta}-\delta_{\alpha \beta} \xi^{\alpha} \otimes \theta^{\beta}$,

where $\varepsilon=\left(\varepsilon_{\alpha \beta}\right)=\left(\begin{array}{cc}I_{p} & 0 \\ 0 & -I_{q}\end{array}\right)$, and $(p, q)$ is the signature of $\Pi$.

We will call $\left(\theta^{\alpha}, \eta, \xi^{\alpha}\right)$ a normalized coframe. Before we prove the theorem, we need the following lemma: 
5.5 Lemma. The set of $n \times n$ matrices $g l(n, \mathbb{R})$ can be decomposed as

$$
g l(n, \mathbb{R})=o(p, q) \oplus o(p, q)^{\perp},
$$

where $o(p, q)^{\perp}=\left\{a \in g l(n, \mathbb{R}) \mid a-\varepsilon a^{t} \varepsilon=0\right\}$.

Proof. Note that any element $a \in g l(n, \mathbb{R})$ can be written as $a=$ $\frac{1}{2}\left(a-\varepsilon a^{t} \varepsilon\right)+\frac{1}{2}\left(a+\varepsilon a^{t} \varepsilon\right)$. From direct computation, it can be easily checked that $\frac{1}{2}\left(a-\varepsilon a^{t} \varepsilon\right) \in o(p, q)$ and $\frac{1}{2}\left(a+\varepsilon a^{t} \varepsilon\right) \in o(p, q)^{\perp}$. Also, it is clear that $o(p, q) \cap o(p, q)^{\perp}=0$, and thus, we have the decomposition.

Remark. We will often use the fact that $a \in o(p, q)^{\perp}$ if and only if $\varepsilon a$ is symmetric, and $a \in o(p, q)$ if and only if $\varepsilon a$ is skew-symmetric.

Proof of Theorem 5.4. First, choose a frame $X_{\alpha} \in \Gamma(L)$ such that $\Pi\left(X_{\alpha}, X_{\beta}\right)=$ $\varepsilon_{\alpha \beta}$ and let $\theta^{\alpha}=-\Phi\left(X_{\alpha}\right)$. Then, let $\tilde{\xi}^{\alpha}$ be 1-forms such that $d \eta=\delta_{\alpha \beta} \theta^{\alpha} \wedge \tilde{\xi}^{\beta}$. (The existence of such $\tilde{\xi}^{\alpha}$ can be checked by using the coordinate functions $\left(x^{i}, y^{j}\right)$ in the normal form theorem.) Note that $\tilde{\xi}^{\alpha}\left(X_{\beta}\right)=\delta_{\beta}^{\alpha}$ since $\theta^{\alpha}=$ $-\Phi\left(X_{\alpha}\right)=-i\left(X_{\alpha}\right) d \eta=\delta_{\gamma \beta} \tilde{\xi}^{\beta}\left(X_{\alpha}\right) \theta^{\gamma}$.

To define $\xi^{\alpha}$ satisfying (2)-(4), consider the following. Note that $\eta, \theta^{\alpha}$ defines an involutive distribution $L$. By the Frobenius Theorem and the computation $d \theta^{\alpha}\left(X_{\beta}, \mathbf{X}_{\eta}\right)=\left\{i\left(X_{\beta}\right) d \theta^{\alpha}\right\}\left(\mathbf{X}_{\eta}\right)=-\left\{\mathscr{L}_{X_{\beta}} \Phi\left(X_{\alpha}\right)\right\}\left(\mathbf{X}_{\eta}\right)=-\left\{\mathscr{L}_{X_{\beta}} \mathscr{L}_{X_{\alpha}} \eta\right\}\left(\mathbf{X}_{\eta}\right)$ $=\Pi\left(X_{\beta}, X_{\alpha}\right)=\varepsilon_{\alpha \beta}$, the exterior derivative of $\theta^{\alpha}$ can be written in the form

$$
d \theta^{\alpha}=\theta^{\beta} \wedge \tilde{\mathbf{a}}_{\beta}^{\alpha}-\varepsilon_{\beta}^{\alpha} \eta \wedge \tilde{\xi}^{\beta} .
$$

where $\varepsilon_{\beta}^{\alpha}$ denotes $\delta^{\alpha \gamma} \varepsilon_{\gamma \beta}$. Let $\left(H_{\beta}^{\alpha} \eta+\tau_{\beta}^{\alpha}\right)$ is the $o(p, q)^{\perp}$-component of $\left(\tilde{\mathbf{a}}_{\beta}^{\alpha}\right)$ with respect to the decomposition $g l(n, \mathbb{R})=o(p, q) \oplus o(p, q)^{\perp}$ where $\tau_{\beta}^{\alpha} \equiv 0 \bmod \theta^{\alpha}, \tilde{\xi}^{\alpha}$.

We claim that $\tilde{\xi}^{\alpha}$ can be chosen so that $H_{\beta}^{\alpha}=0$. More specifically, define $\xi^{\alpha}=\tilde{\xi}^{\alpha}+\varepsilon_{\beta}^{\alpha} H_{\gamma}^{\beta} \theta^{\gamma}$. Then, if we let $\mathbf{a}_{\beta}^{\alpha}=\tilde{\mathbf{a}}_{\beta}^{\alpha}-H_{\beta}^{\alpha} \eta$, we can rewrite equation (5.6) as

$$
d \theta^{\alpha}=\theta^{\beta} \wedge \mathbf{a}_{\beta}^{\alpha}-\varepsilon_{\beta}^{\alpha} \eta \wedge \xi^{\beta} .
$$

(i) We show identity (2) as follows: Since $\varepsilon_{\beta}^{\alpha} H_{\gamma}^{\beta}$ is symmetric $\alpha, \gamma$,

$$
d \eta=\delta_{\alpha \beta} \theta^{\alpha} \wedge \tilde{\xi}^{\beta}=\delta_{\alpha \beta} \theta^{\alpha} \wedge \xi^{\beta}-\varepsilon_{\alpha \beta} H_{\gamma}^{\beta} \theta^{\alpha} \wedge \theta^{\gamma}=\delta_{\alpha \beta} \theta^{\alpha} \wedge \xi^{\beta} .
$$

(ii) To prove (3), it suffices to show that $\lambda^{*} \xi^{\alpha}=\varepsilon_{\beta}^{\alpha} \theta^{\beta}$ since $\Pi$ is the restriction to $L$ of $\varepsilon_{\alpha \beta} \xi^{\alpha} \otimes \xi^{\beta}$. Using the identity (5.3),

$$
\varepsilon_{\beta}^{\alpha}\left(\lambda^{*} \xi^{\alpha}\right)(X)=\varepsilon_{\beta}^{\alpha} \xi^{\alpha}(\lambda(X))=\Pi\left(\lambda(X), X_{\beta}\right)=d \eta\left(X, X_{\beta}\right)=\theta^{\beta}(X) .
$$

(iii) To prove (4), note that $\mathbf{a}_{\beta}^{\alpha}-\tau_{\beta}^{\alpha}$ is an $o(p, q)$-valued 1-form and $\tau_{\beta}^{\alpha} \equiv 0$ $\bmod \theta^{\alpha}, \xi^{\beta}$. If we rewrite the identity (5.7) as $d \theta^{\alpha}=-\left(\mathbf{a}_{\beta}^{\alpha}-\tau_{\beta}^{\alpha}\right) \wedge \theta^{\beta}-\varepsilon_{\beta}^{\alpha} \eta \wedge$ $\xi^{\beta}-\tau_{\beta}^{\alpha} \wedge \theta^{\beta}$, we have $\mathscr{L}_{\mathbf{X}_{\eta}} \theta^{\alpha}=i\left(\mathbf{X}_{\eta}\right) d \theta^{\alpha}=-\left(\mathbf{a}_{\beta}^{\alpha}-\tau_{\beta}^{\alpha}\right)\left(\mathbf{X}_{\eta}\right) \theta^{\beta}-\varepsilon_{\beta}^{\alpha} \xi^{\beta}$. Using 
this and the fact that $\mathbf{a}_{\beta}^{\alpha}-\tau_{\beta}^{\alpha}$ is an $o(p, q)$-valued 1-form,

$$
\begin{aligned}
\mathscr{L}_{\mathbf{x}_{\eta}} \widetilde{\boldsymbol{\Pi}} & =\mathscr{L}_{\mathbf{x}_{\eta}}\left(\varepsilon_{\alpha \beta} \theta^{\alpha} \otimes \theta^{\beta}\right)=\varepsilon_{\alpha \beta}\left\{\left(\mathscr{L}_{\mathbf{x}_{\eta}} \theta^{\alpha}\right) \otimes \theta^{\beta}+\theta^{\alpha} \otimes\left(\mathscr{L}_{\mathbf{x}_{\eta}} \theta^{\beta}\right)\right\} \\
& =-\delta_{\alpha \beta} \xi^{\alpha} \otimes \theta^{\beta}-\delta_{\alpha \beta} \theta^{\alpha} \otimes \xi^{\beta} .
\end{aligned}
$$

It can be easily seen that the normalized coframes in Theorem 5.4 satisfy the transformation rule of the form

$$
\tilde{\theta}^{\alpha}=A_{\beta}^{\alpha} \theta^{\beta}, \quad \tilde{\eta}=\eta, \quad\left(A^{t}\right)_{\beta}^{\alpha} \xi^{\beta}=\tilde{\xi}^{\alpha}, \quad \text { where }\left(A_{\beta}^{\alpha}\right) \in O(p, q) .
$$

In particular, there is a canonical splitting of the short exact sequence

$$
0 \longrightarrow Q^{*} \longrightarrow \operatorname{Ann}\left(\mathbf{X}_{\eta}\right) \longrightarrow L^{*} \longrightarrow 0
$$

defined by a map from $L^{*}$ to $\operatorname{Ann}\left(\mathbf{X}_{\eta}\right)$ sending $\xi_{\mid L}^{\alpha}$ to $\xi^{\alpha}$. Equivalently, there is a canonical decomposition $T^{*} P=E^{*} \oplus Q^{*} \oplus L^{*}$ where $L^{*}$ is spanned by $\xi^{\alpha}$. Also, we have $T P=N \oplus L=E \oplus Q \oplus L$ where $E$ is the line bundle spanned by $\mathbf{X}_{\eta}, N=\operatorname{Ann}\left(L^{*}\right)$, and $Q=\operatorname{Ann}(\eta) \cap \operatorname{Ann}\left(L^{*}\right)$. With respect to this decomposition, $\wedge^{k} L^{*}$ can be regarded as subbundle of the bundle $\wedge^{k} T^{*} P$, and $\boldsymbol{\Pi}$ and $\mathbf{G}$ are symmetric forms on $P$ :

$$
\boldsymbol{\Pi}=\varepsilon_{\alpha \beta} \xi^{\alpha} \otimes \xi^{\beta} \quad \text { and } \quad \mathbf{G}=G_{\alpha \beta \gamma} \xi^{\alpha} \otimes \xi^{\beta} \otimes \xi^{\gamma} .
$$

5.9 The canonical connection on $P$. Nondegenerate Legendre foliations have a canonical connection characterized by the properties in the following theorem.

5.10 Theorem. There is unique connection $\nabla$ on $P$ such that

(1) $\nabla \eta=\nabla(d \eta)=\nabla \boldsymbol{\Pi}=\nabla \widetilde{\mathbf{\Pi}}=\nabla\left(\mathscr{L}_{\mathbf{x}_{\eta}} \widetilde{\mathbf{\Pi}}\right)=0$,

(2) The torsion $\mathbf{T}$ of $\nabla$ has vanishing component in $Q \otimes\left(\bigwedge^{2} N^{*}\right)$ and $L \otimes\left(\bigwedge^{2} L^{*}\right)$.

Remark. To show the existence of $\nabla$, we explicitly give a formula for $\nabla$ using Lie derivatives. This formula is analogous to an expression of the Levi-Civita connection on a Riemannian manifold $(M, g)$ written as follows: $\nabla_{X} v=$ $\frac{1}{2}\left\{i(X)\left(d v+\mathscr{L}_{v^{*}} g\right)\right\}$ where $v^{*}$ is the vector field determined by the condition $g\left(v^{*}, Y\right)=v(Y)$ for all $Y$ where $X$ and $v$ are a vector field and a 1 -form on $M$. It can be easily checked that this formula defines a well-defined connection on $M$, and that it is the Levi-Civita connection.

Proof of Theorem 5.10. Define the natural connection $\nabla$ on $P$ as follows: Let $v \in \Gamma\left(Q^{*}\right)$ and $X$ be a vector field on $P$. Since $\widetilde{\boldsymbol{\Pi}}$ is nondegenerate on $Q, v$ determines a unique section $v^{*}$ of $Q$ by $\widetilde{\boldsymbol{\Pi}}\left(v^{*}, Y\right)=v(Y)$ for all $Y \in \Gamma(Q)$. Now, let

$$
\nabla_{X} v=\frac{1}{2} \pi\left\{i(X)\left(d v+\mathscr{L}_{v^{*}} \cdot \widetilde{\mathbf{\Pi}}\right)\right\}
$$

where $\pi: T^{*} P \rightarrow Q^{*}$ is the projection onto $Q^{*}$ with respect to the canonical decomposition of $T^{*} P$ described above. To extend $\nabla$ to $T^{*} P$, define $\nabla_{X} v$ 
for $v \in \Gamma\left(E^{*}\right)$ and $v \in \Gamma\left(L^{*}\right)$ as follows. If $v \in \Gamma\left(E^{*}\right)$, let $\nabla_{X} v=0$. To define $\nabla_{X} v$ for $v \in \Gamma\left(L^{*}\right)$, note that $\operatorname{ker}(\lambda)=E \oplus L$ and hence the dual bundle map $\lambda^{*}$ of $\lambda$ maps $L^{*}$ isomorphically onto $Q^{*}$. Let $\nabla_{X} v=\left(\lambda^{*}\right)^{-1}\left\{\nabla_{X} \lambda^{*} v\right\}$. We claim that this is the connection on $P$ satisfying the properties (1)-(2) in Theorem 5.10.

To prove the theorem, we must show (i) that $\nabla$ is a well-defined connection on $P$, (ii) that $\nabla$ satisfies the condition (1), (iii) that the $Q \otimes\left(\bigwedge^{2} N^{*}\right)$ component of $\mathbf{T}$ vanishes, (iv) that the $L \otimes\left(\bigwedge^{2} L^{*}\right)$-component of $\mathbf{T}$ vanishes, and $(v)$ the uniqueness of such connection.

(i) To show that $\nabla$ is well-defined, it suffices to show that the identity (5.11) defines a well-defined connection on $Q^{*}$. Clearly, $\nabla_{X} v$ is linear in $X$ and hence we only need prove that $\nabla_{X}$ is a derivation. From direct computation, we have $\mathscr{L}_{f v^{*}} \theta^{\alpha}=\theta^{\alpha}\left(v^{*}\right) d f+f \mathscr{L}_{v^{*}} \theta^{\alpha}$. Thus, using the identity $\widetilde{\boldsymbol{\Pi}}=\varepsilon_{\alpha \beta} \theta^{\alpha} \otimes \theta^{\beta}$, we obtain $i(X) \mathscr{L}_{f v^{*}} \widetilde{\boldsymbol{\Pi}}=(X f) v+v(X) d f+f i(X) \mathscr{L}_{v^{*}} \widetilde{\boldsymbol{\Pi}}$. Substituting this to the identity (5.11), proves that $\nabla_{X}$ is a derivation:

$$
\nabla_{X} f v=\frac{1}{2} \pi\left\{i(X)\left(d(f v)+\mathscr{L}_{f v^{*}} \tilde{\boldsymbol{\Pi}}\right)\right\}=(X f) v+f \nabla_{X} v .
$$

(ii) To prove property (1), we will show that there is an $o(p, q)$-valued 1form $\left(\pi_{\beta}^{\alpha}\right)$ such that

$$
\nabla \theta^{\alpha}=-\pi_{\beta}^{\alpha} \otimes \theta^{\beta}, \quad \nabla \xi^{\alpha}=\left(\pi^{t}\right)_{\beta}^{\alpha} \otimes \xi^{\beta} .
$$

where $\left(\theta^{\alpha}, \eta, \xi^{\alpha}\right)$ is the normalized coframe in Theorem 5.4. From this, property (1) easily follows. For instance,

$$
\begin{aligned}
\nabla_{X} d \eta & =\nabla_{X}\left(\delta_{\alpha \beta} \theta^{\alpha} \wedge \xi^{\beta}\right) \\
& =\delta_{\alpha \beta} \pi_{\gamma}^{\alpha}(X) \theta^{\gamma} \wedge \xi^{\beta}-\delta_{\alpha \beta} \theta^{\alpha} \wedge\left\{\left(\pi^{t}\right)_{\gamma}^{\beta}(X) \xi^{\gamma}\right\}=0 .
\end{aligned}
$$

Other properties can be proved similarly.

To construct $\pi_{\beta}^{\alpha}$ and prove property (2), it is convenient to consider the following formula, which will simplify the computation: Let $W_{\alpha}, \mathbf{x}_{\eta}$, and $X_{\alpha}$ be the dual frame of $\theta^{\alpha}, \eta$, and $\xi^{\alpha}$, then $\left(\theta^{\alpha}\right)^{*}=\varepsilon^{\alpha \nu} W_{\nu}$ and $\left(\nabla_{X} \theta^{\alpha}\right)(Y)=$ $\frac{1}{2}\left\{d \theta^{\alpha}(X, Y)+\varepsilon^{\alpha \nu} \mathscr{L}_{W_{\nu}}\left(\varepsilon_{\sigma \rho} \theta^{\sigma} \otimes \theta^{\rho}\right)(X, Y)\right\}$ from (5.11). From this, we obtain the formula

$$
\begin{aligned}
\left(\nabla_{X} \theta^{\alpha}\right)(Y)=\frac{1}{2}\left\{d \theta^{\alpha}(X, Y)-\varepsilon^{\alpha \nu} d \theta^{\sigma}\left(X, W_{\nu}\right) \varepsilon_{\sigma \rho} \theta^{\rho}(Y)\right. \\
\left.+\varepsilon_{\sigma \rho} \theta^{\sigma}(X) \varepsilon^{\alpha \nu} d \theta^{\rho}\left(W_{\nu}, Y\right)\right\} .
\end{aligned}
$$

Letting $Y=W_{\beta}$ in (5.13), we obtain the formula for $\left(\pi_{\beta}^{\alpha}\right)$

$$
\begin{aligned}
\pi_{\beta}^{\alpha}(X)=- & \left(\nabla_{X} \theta^{\alpha}\right)\left(W_{\beta}\right) \\
=- & \frac{1}{2}\left\{d \theta^{\alpha}\left(X, W_{\beta}\right)-\varepsilon^{\alpha \nu} d \theta^{\sigma}\left(X, W_{\nu}\right) \varepsilon_{\sigma \beta}\right\} \\
& +\frac{1}{2} \varepsilon_{\sigma \rho} \theta^{\sigma}(X)\left\{\varepsilon^{\alpha \nu} d \theta^{\rho}\left(W_{\nu}, W_{\beta}\right)\right\} .
\end{aligned}
$$


Recall that $a \in g l(n, \mathbb{R})$ is in $o(p, q)$ if and only if $\varepsilon a$ is skew-symmetric. Hence, it follows that the last two terms in (5.14) are $o(p, q)$-valued as matrices in $\alpha, \beta$, and therefore, so is $\pi_{\beta}^{\alpha}$. Also, it can be directly computed that $\lambda^{*} \xi^{\alpha}=$ $\varepsilon_{\beta}^{\alpha} \theta^{\alpha}$, and

$$
\nabla_{X} \xi^{\alpha}=\left(\lambda^{*}\right)^{-1}\left\{\nabla_{X} \lambda^{*} \xi^{\alpha}\right\}=-\varepsilon_{\beta}^{\alpha} \pi_{\gamma}^{\beta}(X) \varepsilon_{\sigma}^{\gamma} \xi^{\sigma}=\left(\pi^{t}\right)_{\beta}^{\alpha}(X) \xi^{\beta} .
$$

(iii) To prove that $\mathbf{T}$ has vanishing component in $Q \otimes\left(\bigwedge^{2} N^{*}\right)$, we need to show

$$
\theta^{\alpha}\{\mathbf{T}(X, Y)\}=d \theta^{\alpha}(X, Y)-\left(\nabla_{X} \theta^{\alpha}\right)(Y)+\left(\nabla_{Y} \theta^{\alpha}\right)(X)=0 .
$$

for $X, Y$ are sections of $N$. But this can be easily seen by considering the skew symmetric part in $X, Y$ of the formula (5.13).

(iv) To prove that $\mathbf{T}$ has vanishing $L \otimes\left(\bigwedge^{2} L^{*}\right)$-component, we need to show

$$
\xi^{\alpha}\left\{\mathbf{T}\left(X_{\beta}, X_{\gamma}\right)\right\}=d \xi^{\alpha}\left(X_{\beta}, X_{\gamma}\right)-\left(\pi^{t}\right)_{\gamma}^{\alpha}\left(X_{\beta}\right)+\left(\pi^{t}\right)_{\beta}^{\alpha}\left(X_{\gamma}\right)=0 .
$$

The proof of this makes use of the following three identities. First, let $X=X_{\gamma}$, $Y=W_{\beta}$, and substitute (5.7) in the formula (5.13). Then

$$
\pi_{\beta}^{\alpha}\left(X_{\gamma}\right)=-\left(\nabla_{X_{\gamma}} \theta^{\alpha}\right)\left(W_{\beta}\right)=\frac{1}{2}\left\{\mathbf{a}_{\beta}^{\alpha}\left(X_{\gamma}\right)-\varepsilon^{\alpha \nu} \varepsilon_{\beta \sigma} \mathbf{a}_{\nu}^{\sigma}\left(X_{\gamma}\right)\right\} .
$$

We obtain the second identity from $0=d^{2} \eta=\delta_{\sigma \nu} d \theta^{\sigma} \wedge \xi^{\nu}-\delta_{\sigma \nu} \theta^{\sigma} \wedge d \xi^{\nu}$. From this and the identity (5.7), $\delta_{\sigma \nu} \mathbf{a}_{\rho}^{\sigma} \wedge \xi^{\nu} \wedge \theta^{\rho}=\delta_{\sigma \nu} d \xi^{\sigma} \wedge \theta^{\nu}$. Applying interior evaluation with $X_{\beta} \wedge X_{\gamma} \wedge W_{\sigma}$ on both sides of this identity, we obtain an identity

$$
\frac{1}{2} d \xi^{\alpha}\left(X_{\beta}, X_{\gamma}\right)=\frac{1}{2}\left\{\mathbf{a}_{\alpha}^{\gamma}\left(X_{\beta}\right)-\mathbf{a}_{\alpha}^{\beta}\left(X_{\gamma}\right)\right\} .
$$

To obtain the last identity, note that $d \theta^{\sigma}\left(X_{\beta}, X_{\sigma}\right)=0$ from the involutivity of $L$. Differentiating this by $\mathscr{L}_{\mathbf{X}_{\eta}}$,

$$
\begin{aligned}
0 & =\mathscr{L}_{\mathbf{X}_{\eta}}\left\{\varepsilon_{\sigma}^{\alpha} d \theta^{\sigma}\left(X_{\beta}, X_{\sigma}\right)\right\} \\
& =\varepsilon_{\sigma}^{\alpha}\left\{\left(\mathscr{L}_{\mathbf{x}_{\eta}} d \theta^{\sigma}\right)\left(X_{\beta}, X_{\gamma}\right)+d \theta^{\sigma}\left(\mathscr{L}_{\mathbf{x}_{\eta}} X_{\beta}, X_{\gamma}\right)+d \theta^{\gamma}\left(X_{\beta}, \mathscr{L}_{\mathbf{x}_{\eta}} X_{\gamma}\right)\right\} .
\end{aligned}
$$

Using (5.7) and $\eta\left(\mathscr{L}_{\mathbf{x}_{\eta}} X_{\beta}\right)=0$, we compute

$$
\begin{aligned}
d \theta^{\alpha}\left(\mathscr{L}_{\mathbf{X}_{\eta}} X_{\beta}, X_{\gamma}\right) & =\theta^{\sigma}\left(\mathscr{L}_{\mathbf{X}_{\eta}} X_{\beta}\right) \mathbf{a}_{\sigma}^{\alpha}\left(X_{\gamma}\right)=\varepsilon_{\beta}^{\sigma} \mathbf{a}_{\sigma}^{\alpha}\left(X_{\gamma}\right)\left(\mathscr{L}_{\mathbf{X}_{\eta}} d \theta^{\sigma}\right)\left(X_{\beta}, X_{\gamma}\right) \\
& =d\left\{i\left(\mathbf{X}_{\eta}\right) d \theta^{\sigma}\right\}\left(X_{\beta}, X_{\gamma}\right)=-\varepsilon_{\rho}^{\sigma} d \xi^{\rho}\left(X_{\beta}, X_{\gamma}\right) .
\end{aligned}
$$

Hence, the last identity follows:

$$
\frac{1}{2} d \xi^{\alpha}\left(X_{\beta}, X_{\gamma}\right)=\frac{1}{2}\left\{\varepsilon_{\sigma}^{\alpha} \varepsilon_{\beta}^{\rho} \mathbf{a}_{\rho}^{\sigma}\left(X_{\gamma}\right)-\varepsilon_{\sigma}^{\alpha} \varepsilon_{\gamma}^{\rho} \mathbf{a}_{\rho}^{\sigma}\left(X_{\beta}\right)\right\} .
$$

Adding (5.18) and (5.19), we obtain an expression for $d \xi^{\alpha}\left(X_{\beta}, X_{\gamma}\right)$ in terms of $\mathbf{a}_{\beta}^{\alpha}$. The identity (5.16) can be checked by direct computation using this expression and equation (5.17). 
(v) For uniqueness, suppose that $\tilde{\nabla}$ is another such connection satisfying conditions (1) and (2). Then $0=\widetilde{\nabla}_{X}\left\{d \eta-\mathscr{L}_{\mathbf{X}_{\eta}} \widetilde{\mathbf{I}}\right\}=\widetilde{\nabla}_{X}\left\{\delta_{\alpha \beta} \theta^{\alpha} \otimes \xi^{\beta}\right\}$, which implies that $\tilde{\nabla}$ has connection form $\tilde{\pi}_{\beta}^{\alpha}$ such that $\tilde{\nabla} \theta^{\alpha}=-\tilde{\pi}_{\beta}^{\alpha} \otimes \theta^{\beta}$, and $\tilde{\nabla} \xi^{\alpha}=\left(\tilde{\pi}^{t}\right)_{\beta}^{\alpha} \otimes \xi^{\beta}$. Moreover, it can be easily checked that $\tilde{\nabla} \widetilde{\boldsymbol{\Pi}}=0$ implies that $\tilde{\pi}_{\beta}^{\alpha}$ is an $o(p, q)$-valued 1 -form. Now, since the torsions $\mathbf{T}$ and $\widetilde{\mathbf{T}}$ have vanishing $Q \otimes\left(\wedge^{2} N^{*}\right)$-component, we have

$$
\begin{aligned}
& \theta^{\alpha}\{\mathbf{T}(X, Y)\}=d \theta^{\alpha}(X, Y)+\pi_{\gamma}^{\alpha}(X) \theta^{\gamma}(Y)-\pi_{\gamma}^{\alpha}(Y) \theta^{\gamma}(X)=0, \\
& \theta^{\alpha}\{\widetilde{\mathbf{T}}(X, Y)\}=d \theta^{\alpha}(X, Y)+\tilde{\pi}_{\gamma}^{\alpha}(X) \theta^{\gamma}(Y)-\tilde{\pi}_{\gamma}^{\alpha}(Y) \theta^{\gamma}(X)=0,
\end{aligned}
$$

where $X, Y$ are sections of $N$. By letting $X=\mathbf{X}_{\eta}$ and comparing both equations, we obtain $\pi_{\beta}^{\alpha}\left(\mathbf{X}_{\eta}\right)=\tilde{\pi}_{\beta}^{\alpha}\left(\mathbf{X}_{\eta}\right)$. If we let $X=W_{\sigma}$ and $Y=W_{\rho}$, it follows that $\pi_{\rho}^{\alpha}\left(W_{\sigma}\right)-\tilde{\pi}_{\rho}^{\alpha}\left(W_{\sigma}\right)$ is symmetric in $\rho, \sigma$ by comparing both equations again. But, since $\pi_{\beta}^{\alpha}$, and $\tilde{\pi}_{\beta}^{\alpha}$ are $o(p, q)$-valued 1-forms, $\varepsilon_{\beta \alpha}\left\{\pi_{\rho}^{\alpha}\left(W_{\sigma}\right)-\tilde{\pi}_{\rho}^{\alpha}\left(W_{\sigma}\right)\right\}$ vanishes since it is symmetric in $\rho, \sigma$, and skew symmetric in $\beta, \rho$. It follows that $\pi_{\beta}^{\alpha}$, and $\tilde{\pi}_{\beta}^{\alpha}$ coincide in the direction of $\operatorname{Ann}\left(L^{*}\right)$. A similar argument using the fact that both $\mathbf{T}$ and $\widetilde{\mathbf{T}}$ have vanishing $L \otimes\left(\bigwedge^{2} L^{*}\right)$-component (identity (5.16)) shows that $\pi_{\beta}^{\alpha}$ and $\tilde{\pi}_{\beta}^{\alpha}$ coincide in the direction of $L$.

5.20 Theorem. $\theta^{\alpha}, \eta, \xi^{\alpha}$, and $\pi_{\beta}^{\alpha}$ satisfy the first structure equations

$$
\begin{aligned}
\left(\begin{array}{c}
d \theta^{\alpha} \\
d \eta \\
d \xi^{\alpha}
\end{array}\right)= & -\left(\begin{array}{ccc}
\pi_{\beta}^{\alpha} & 0 & 0 \\
0 & 0 & 0 \\
0 & 0 & -\left(\pi^{t}\right)_{\beta}^{\alpha}
\end{array}\right)\left(\begin{array}{c}
\theta^{\beta} \\
\eta \\
\xi^{\beta}
\end{array}\right) \\
& +\left(\begin{array}{c}
-\varepsilon_{\beta}^{\alpha} \eta \wedge \xi^{\beta}+G^{\alpha}{ }_{\sigma \gamma} \varepsilon_{\beta}^{\sigma} \theta^{\beta} \wedge \xi^{\gamma} \\
\delta_{\beta \gamma} \theta^{\beta} \wedge \xi^{\gamma} \\
K_{\beta \gamma}^{\alpha} \theta^{\beta} \wedge \theta^{\gamma}+S_{\beta}^{\alpha} \eta \wedge \theta^{\beta}+Q^{\alpha}{ }_{\beta \gamma} \theta^{\beta} \wedge \xi^{\gamma}
\end{array}\right),
\end{aligned}
$$

where $Q_{\beta \gamma}^{\alpha}=Q_{\alpha \gamma}^{\beta}$ and $K_{\beta \gamma}^{\alpha}=-K_{\gamma \beta}^{\alpha} \cdot\left(G_{\beta \gamma}^{\alpha}\right.$ denotes $\left.\delta^{\alpha \sigma} G_{\sigma \beta \gamma}.\right)$

Proof. The first equation comes from the identity (5.7): If we let $\mathbf{a}_{\beta}^{\alpha}-\pi_{\beta}^{\alpha}=$ $C_{\beta \gamma}^{\alpha} \theta^{\gamma}+D_{\beta \gamma}^{\alpha} \xi^{\gamma}$,

$$
d \theta^{\alpha}=-\pi_{\beta}^{\alpha} \wedge \theta^{\beta}-\varepsilon_{\beta}^{\alpha} \eta \wedge \xi^{\beta}+C_{\beta \gamma}^{\alpha} \theta^{\beta} \wedge \theta^{\gamma}+D_{\beta \gamma}^{\alpha} \theta^{\beta} \wedge \xi^{\gamma} .
$$

By substituting this into (5.15), and letting $X=W_{\alpha}, Y=W_{\beta}$, we obtain $C_{\beta \gamma}^{\alpha}=0$. Therefore, $\mathbf{a}_{\beta}^{\alpha}=\pi_{\beta}^{\alpha}+D^{\alpha}{ }_{\beta \gamma} \xi^{\gamma}$ and by substituting this into the identity (5.17), we can check that $D^{\alpha}{ }_{\beta \gamma}$ is $o(p, q)^{\perp}$-valued as a matrix in $\alpha, \beta$ for fixed $\gamma$.

We claim $D_{\beta \gamma}^{\alpha}=\varepsilon_{\beta}^{\sigma} G_{\gamma \sigma}^{\alpha}$. To do this, we use the formula for $\mathbf{G}$ given in Proposition 3.19. Using equation (5.22) above, we compute $\nabla_{X_{\gamma}} \theta^{\alpha}=\mathscr{L}_{X_{\gamma}} \theta^{\alpha}=$ $-\left\{\pi_{\beta}^{\alpha}\left(X_{\gamma}\right)+D^{\alpha}{ }_{\beta \gamma}\right\} \theta^{\beta}-\varepsilon_{\beta}^{\alpha} \eta$. Thus $c^{a \beta}=\varepsilon^{\alpha \beta}$, and $G_{\alpha \beta \gamma}$ is the symmetric part of $\varepsilon^{\gamma \rho} \omega_{\rho}^{\beta}\left(X_{\alpha}\right)$ in $\beta, \gamma$. Since $\omega_{\rho}^{\beta}\left(X_{\alpha}\right) \theta^{\rho}=\left\{\pi_{\rho}^{\beta}\left(X_{\alpha}\right)+D_{\rho \alpha}^{\beta}\right\} \theta^{\rho}$ and $D_{\rho \alpha}^{\beta}$ is 
$o(p, q)^{\perp}$-valued in $\beta, \rho, G_{\alpha \beta \gamma}=\varepsilon_{\gamma}^{\rho} D_{\rho \alpha}^{\beta}$. From this and the symmetry of $G_{\alpha \beta \gamma}$, the claim follows.

We complete the proof by showing that the coefficients of $\xi^{\beta} \wedge \xi^{\gamma}$ and $\eta \wedge \xi^{\beta}$ in $d \xi^{\alpha}-\left(\pi^{t}\right)_{\beta}^{\alpha} \wedge \xi^{\beta}$ vanish. The vanishing of the coefficient of $\xi^{\beta} \wedge \xi^{\gamma}$ follows from the identity (5.16). The vanishing of the coefficient of $\eta \wedge \xi^{\beta}$ and the symmetry condition on $Q_{\beta \gamma}^{\alpha}$ can be shown by evaluating both sides of $0=$ $d^{2} \theta=\delta_{\sigma \rho}\left(d \theta^{\sigma} \wedge \xi^{\rho}-\theta^{\sigma} \wedge d \xi^{\rho}\right)$ by $\mathbf{X}_{\eta} \wedge X_{\gamma} \wedge W_{\alpha}$ and $W_{\alpha} \wedge W_{\beta} \wedge X_{\gamma}$.

Recall that $\mathbf{G}=G_{\alpha \beta \gamma} \xi^{\alpha} \otimes \xi^{\beta} \otimes \xi^{\gamma}$.

5.23 Proposition. The invariant $\mathbf{G}$ satisfies the identity

$$
\nabla_{\mathbf{x}_{\eta}} \mathbf{G}=-\varepsilon_{\alpha}^{\beta} \varepsilon_{\sigma}^{\gamma} Q_{\beta \gamma \rho} \xi^{\alpha} \otimes \xi^{\sigma} \otimes \xi^{\rho}
$$

Moreover, $Q_{\beta \gamma}^{\alpha}$ is an $o(p, q)^{\perp}$-valued function as a matrix in $\alpha, \gamma$ and $\beta, \gamma$. In particular, if $\mathbf{G}=0$, then $Q_{\beta \gamma}^{\alpha}=0$.

Proof. This follows from the evaluation of both sides of $d^{2} \theta^{\alpha}=0$ by $W_{\sigma} \wedge$ $\mathbf{X}_{\eta} \wedge X_{\rho}$. For simplicity, let us denote $\mu_{\gamma}^{\alpha}=\varepsilon_{\beta}^{\sigma} G^{\alpha}{ }_{\gamma \sigma} \theta^{\beta}$. Also, let $\theta=\left(\theta^{\alpha}\right)$, $\xi=\left(\xi^{\alpha}\right), \pi=\left(\pi_{\beta}^{\alpha}\right)$, and $\mu=\left(\mu_{\beta}^{\alpha}\right)$. Then, from the structure equation (5.21),

$$
d \theta=-\pi \wedge \theta-(\varepsilon \eta) \wedge \xi+\mu \wedge \xi, \quad d \xi=\pi^{t} \wedge \xi+\kappa,
$$

where $\kappa=\left(\kappa^{\alpha}\right)=K_{\beta \gamma}^{\alpha} \theta^{\beta} \wedge \theta^{\gamma}+S_{\beta}^{\alpha} \eta \wedge \theta^{\beta}+Q_{\beta \gamma}^{\alpha} \theta^{\beta} \wedge \xi^{\gamma}$. Now compute the exterior derivative of the first equation:

$$
\begin{aligned}
0= & d^{2} \theta=-d \pi \wedge \theta+\pi \wedge d \theta-d(\varepsilon \eta-\mu) \wedge \xi+(\varepsilon \eta-\mu) \wedge d \xi \\
= & -(d \pi+\pi \wedge \pi) \wedge \theta-\pi \wedge(\varepsilon \eta-\mu) \wedge \xi-d(\varepsilon \eta-\mu) \wedge \xi \\
& +(\varepsilon \eta-\mu) \wedge \pi^{t} \wedge \xi+(\varepsilon \eta-\mu) \wedge \kappa \\
= & -(d \pi+\pi \wedge \pi) \wedge \theta+(\varepsilon \eta-\mu) \wedge \kappa \\
& -\left\{d(\varepsilon \eta-\mu)+\pi \wedge(\varepsilon \eta-\mu)-(\varepsilon \eta-\mu) \wedge \pi^{t}\right\} \wedge \xi .
\end{aligned}
$$

But, since $\pi$ is an $o(p, q)$-valued 1 -form, we have $\pi \wedge(\varepsilon \eta)-(\varepsilon \eta) \wedge \pi^{t}=0$. Applying interior evaluation with $W_{\sigma} \wedge \mathbf{X}_{\eta} \wedge X_{\rho}$ to both sides of the equation above, we obtain

$$
\begin{aligned}
\{d \pi & +\pi \wedge \pi\}_{\sigma}^{\alpha}\left(\mathbf{X}_{\eta}, X_{\rho}\right) \\
& =\left\{d \mu+\pi \wedge \mu-\mu \wedge \pi^{t}\right\}_{\rho}^{\alpha}\left(W_{\sigma}, \mathbf{X}_{\eta}\right)-\left\{\varepsilon_{\beta}^{\alpha} \kappa^{\beta}\right\}\left(W_{\sigma}, X_{\rho}\right) .
\end{aligned}
$$

Observe that the left-hand side of this identity is $o(p, q)$-valued regarded as matrices in $\alpha, \sigma$. We claim that and the right-hand side is $o(p, q)^{\perp}$-valued in 
$\alpha, \sigma$, and hence it vanishes. From direct computation, we obtain

$$
\begin{aligned}
\{d \mu+ & \left.\pi \wedge \mu-\mu \wedge \pi^{t}\right\}_{\rho}^{\alpha}\left(W_{\sigma}, \mathbf{X}_{\eta}\right) \\
= & -\varepsilon_{\sigma}^{\gamma}\left(\mathbf{X}_{\eta} G_{\rho \gamma}^{\alpha}\right)-\varepsilon_{\sigma}^{\gamma} G_{\beta \gamma}^{\alpha}\left(\pi^{t}\right)_{\rho}^{\beta}\left(\mathbf{X}_{\eta}\right) \\
& -\sum_{\gamma=1}^{n} \varepsilon_{\sigma}^{\gamma}\left(G_{\rho \alpha}^{\beta} \pi_{\beta}^{\gamma}\left(\mathbf{X}_{\eta}\right)+G_{\rho \gamma}^{\beta} \pi_{\beta}^{\alpha}\left(\mathbf{X}_{\eta}\right)\right) .
\end{aligned}
$$

Note that terms on the right-hand side of this equation are components of $o(p, q)^{\perp}$-valued matrices in $\alpha, \sigma$. Also, note the formula $-\left\{\varepsilon_{\beta}^{\alpha} \kappa^{\beta}\right\}\left(W_{\sigma}, X_{\rho}\right)$ $=-\varepsilon_{\beta}^{\alpha} Q_{\sigma \rho}^{\beta}$. Since $Q_{\sigma \rho}^{\beta}$ is symmetric in $\beta, \sigma,-\left\{\varepsilon_{\beta}^{\alpha} \kappa^{\beta}\right\}\left(W_{\sigma}, X_{\rho}\right)$ is an $o(p, q)^{\perp}$-valued function in $\alpha, \sigma$ for each fixed $\rho$, and thus we have proved the claim. Therefore, the right-hand side of the identity (5.24) vanishes. From this, we obtain the formula

$$
\begin{aligned}
-\varepsilon_{\alpha}^{\beta} \varepsilon_{\sigma}^{\gamma} Q_{\beta \gamma \rho}= & \left(\mathbf{X}_{\eta} G_{\alpha \sigma \rho}\right)+G_{\beta \sigma \rho}\left(\pi^{t}\right)_{\alpha}^{\beta}\left(\mathbf{X}_{\eta}\right) \\
& +G_{\alpha \beta \rho}\left(\pi^{t}\right)_{\sigma}^{\beta}\left(\mathbf{X}_{\eta}\right)+G_{\alpha \sigma \beta}\left(\pi^{t}\right)_{\rho}^{\beta}\left(\mathbf{X}_{\eta}\right),
\end{aligned}
$$

and this proves the proposition. Finally, note that the right-hand side of this equation is symmetric in all of $\alpha, \sigma$, and $\rho$. Therefore, $Q_{\beta \gamma}^{\alpha}$ is an $o(p, q)^{\perp}$ valued function regarded as matrices in $\alpha, \gamma$ for fixed $\beta$, and in $\beta, \gamma$ for each fixed $\alpha$.

5.25 $\Pi$ as a pseudo-Riemannian metric on leaves. If $\mathscr{F}$ is nondegenerate, the leaves of $\mathscr{F}$ have a canonical pseudo-Riemannian metric given by the restriction of $\Pi$. Recall that each leaf of $\mathscr{F}$ has a canonical affine connection (§3.22). Similarly, consider the Levi-Civita connection of this pseudo-Riemannian on the leaves. In fact, this connection is the restriction of $\nabla$ to the leaves since the torsion of $\nabla$ has $L \otimes\left(\wedge^{2} L^{*}\right)$-vanishing component and $\nabla_{X} \Pi=0$. We will show that the curvature of the Levi-Civita connection can be expressed in terms of the invariant $\mathbf{G}$. As a corollary, in the special case when $\mathscr{F}$ is Riemannian, the canonical Riemannian metric has positive constant sectional curvature.

To obtain the curvature of the Levi-Civita connection, we compute the curvature of $\nabla$ in directions tangent to $\mathscr{F}$ :

5.26 Proposition. If $\Omega_{\beta}^{\alpha}=d \pi_{\beta}^{\alpha}+\pi_{\gamma}^{\alpha} \wedge \pi_{\beta}^{\gamma}$. is the curvature matrix of $\nabla$,

$$
\Omega_{\beta}^{\alpha} \equiv \varepsilon_{\beta \gamma} \delta_{\rho}^{\alpha} \xi^{\rho} \wedge \xi^{\gamma}+\left(D_{\gamma \rho}^{\beta} \xi^{\rho}\right) \wedge\left(D_{\alpha \sigma}^{\gamma} \xi^{\sigma}\right) \bmod \left(\theta^{a}, \eta\right)
$$

where $D^{\alpha}{ }_{\beta \gamma}=\varepsilon_{\beta}^{\sigma} G^{\alpha}{ }_{\gamma \sigma}$. If $\boldsymbol{\Pi}$ is positive definite and $\mathbf{G}=0$, then the canonical Levi-Civita connection on leaves has the curvature matrix $\frac{1}{2}\left\{\delta_{\sigma}^{\beta} \delta_{\rho}^{\alpha}-\delta_{\rho}^{\beta} \delta_{\sigma}^{\alpha}\right\} \xi^{\rho} \wedge$ $\xi^{\sigma}$, and hence, the leaves of $\mathscr{F}$ have Riemannian metrics of constant sectional curvature 1.

Proof. From the structure equation (5.21), we have

$$
\omega_{\beta}^{\alpha}\left(X_{\gamma}\right)=-\left\{\nabla_{X_{\gamma}}^{Q} \theta^{\alpha}\right\}\left(W_{\beta}\right)=\pi_{\beta}^{\alpha}\left(X_{\gamma}\right)+D_{\beta \gamma}^{\alpha} .
$$


Hence, as relative forms, we have $\omega_{\beta}^{\alpha}=\pi_{\beta}^{\alpha}+D^{\alpha}{ }_{\beta \gamma} \xi^{\gamma}$ and

$$
\begin{aligned}
d_{\mathscr{F}} \omega_{\beta}^{\alpha}+\omega_{\gamma}^{\alpha} \wedge \omega_{\beta}^{\gamma}= & \left(d_{\mathscr{F}} \pi_{\beta}^{\alpha}+d_{\mathscr{F}}\left(D^{\alpha}{ }_{\beta \gamma} \xi^{\gamma}\right)\right) \\
& +\left(\pi_{\gamma}^{\alpha}-D^{\alpha}{ }_{\gamma \rho} \xi^{\rho}\right) \wedge\left(\pi_{\beta}^{\gamma}-D_{\beta \sigma}^{\gamma} \xi^{\sigma}\right) .
\end{aligned}
$$

On the other hand, from the identities (3.14) and $\omega_{n+1}^{\alpha}=c^{\alpha \beta} \omega_{\beta}^{n+1}$, we have

$$
d_{\mathscr{F}} \omega_{\beta}^{\alpha}+\omega_{\gamma}^{\alpha} \wedge \omega_{\beta}^{\gamma}=-c^{\alpha \gamma} \omega_{\gamma}^{n+1} \wedge \omega_{\beta}^{n+1}=-\varepsilon_{\alpha \gamma} \xi^{\gamma} \wedge \xi^{\beta} .
$$

(Note that $\xi^{\alpha}=\omega_{\alpha}^{n+1}$ as relative forms since $\left(\xi^{\alpha}\right)$ and $\left(\omega_{\alpha}^{n+1}\right)$ are both dual frames to $\left(X_{\alpha}\right)$ where $X_{\alpha} \in \Gamma(L)$ are determined by the condition $\Phi\left(X_{\alpha}\right)=$ $-\theta^{\alpha}$.) Comparing the $o(p, q)$-component of (5.27) and (5.28), we obtain the curvature matrix.

5.29 Examples of nondegenerate Legendre foliations. In the normal form theorem, we proved that any Legendre foliation is locally equivalent to one of the form $\mathscr{F}_{F}$. We study Legendre foliations $\mathscr{F}_{F}$ when (i) $F$ is a Finsler metric and (ii) $F$ is the norm induced by a Riemannian metric on $M$. For a Riemannian manifold $(M, g)$, we denote $\mathscr{F}_{g}$ and $\eta_{g}$ for the Riemannian Legendre foliation and the contact 1-form on the unit cotangent bundle $S_{g}^{*} M$ of $M$.

A Finsler metric $F: T^{*} M \rightarrow \mathbb{R}^{+} \cup\{0\}$ is a positively homogeneous function (i.e. $F(t v)=t F(v)$ for $t>0)$ satisfying the triangle inequality $F\left(v_{1}+v_{2}\right) \leq$ $F\left(v_{1}\right)+F\left(v_{2}\right)$. It can be shown that the triangle inequality is equivalent to the convexity of the function $F^{2}$, and this is again equivalent to the condition that $\left(F^{2}\right)_{i j}$ be positive definite on $T^{*} M$. (See [Sp].)

5.30 Proposition. A Legendre foliation $\mathscr{F}$ is locally equivalent to one of the form $\mathscr{F}_{F}$ with $F$ Finsler metric if and only if $\Pi$ is positive definite.

Proof. Note that, since $F$ is homogeneous, $F=F_{i} y^{i}$. By differentiating this again, we obtain $F_{i j} y^{i}=0$, and hence restricted to fibers of $T^{*} M, d F=$ $F_{i} d y^{i}$. Also, on fibers of $S_{F}^{*} M$, we have $F=1$ and $0=d F=F_{i} d y^{i}$. Therefore,

$$
\frac{1}{2}\left(F^{2}\right)_{i j} d y^{i} \otimes d y^{j}=\left\{F_{i} F_{j}+F F_{i j}\right\} d y^{i} \otimes d y^{j}=F_{i j} d y^{i} \otimes d y^{j}=\Pi .
$$

From this, it is clear that, if $F$ is a Finsler metric, then $\Pi$ is positive definite. Conversely, if $\Pi$ is positive definite, $\mathscr{F}$ is locally equivalent to its normal form $\mathscr{F}_{F}$ with $\left(F^{2}\right)_{i j} d y^{i} \otimes d y^{j}$ positive definite, and thus $F$ is a Finsler metric.

5.32 Proposition. A nondegenerate Legendre foliation $\mathscr{F}$ is pseudo-Riemannian if and only if $\mathbf{G}=0$. If the nondegeneracy condition is replaced by the condition that $\Pi$ is positive definite, the pseudo-Riemannian metric is a Riemannian metric. 
Proof. By the Normal Form Theorem 2.5, we may assume that $\mathscr{F}$ is of the form $\mathscr{F}_{F}$. We claim that

$$
\mathbf{G}=\frac{1}{4}\left(F^{2}\right)_{i j k} d y^{i} \otimes d y^{j} \otimes d y^{k} .
$$

The proof of this can be directly checked using the the following facts: $\frac{1}{2}\left(F^{2}\right)_{i j k}$ $=F_{i k} F_{j}+F_{i} F_{j k}+F_{k} F_{i j}+F F_{i j k}$ and $F_{i} d y^{i}=d F=0$ on $S_{F}^{*} M$.

If $\mathscr{F}$ is Riemannian, then $F^{2}=g_{k l}(x) y^{k} y^{l}$, for a metric $g_{k l}$ depending only upon $x=\left(x^{i}\right)$, and it follows that $\left(F^{2}\right)_{i j k}=0$ and $\mathbf{G}=0$.

Conversely, if $\mathbf{G}=0$ and $\Pi$ is nondegenerate, $\left(F^{2}\right)_{i j k} d y^{i} \otimes d y^{j} \otimes d y^{k}=0$ in the direction tangent to $\mathscr{F}_{F}$. Thus, it suffices to show that $\left(F^{2}\right)_{i j k} d y^{i} \otimes d y^{j} \otimes$ $d y^{k}=0$ in the direction of $y^{i} \frac{\partial}{\partial y^{i}}$, which is transversal to the hypersurface $\{F=$ constant $\}$. (See the proof of Theorem 4.1.) Recall that we have $F_{i} y^{i}=$ $F$ and $F_{i j} y^{j}=0$. By differentiating this with respect to $y^{k}$, we obtain $F_{i j k} y^{j}=-F_{i k}$. Following computation shows that $\left(F^{2}\right)_{i j k} d y^{i} \otimes d y^{j} \otimes d y^{k}=0$ vanishes in the direction of $y^{i} \frac{\partial}{\partial y^{i}}$ :

$$
\begin{aligned}
\frac{1}{2}\left(F^{2}\right)_{i j k} y^{k} & =\left\{F_{i k} F_{j}+F_{j k} F_{i}+F_{i j} F_{k}+F F_{i j k}\right\} y^{k} \\
& =F_{i j} F+F F_{i j k} y^{k}=F_{i j} F-F_{i j} F=0 .
\end{aligned}
$$

Therefore, $\left(F^{2}\right)_{i j k} d y^{i} \otimes d y^{j} \otimes d y^{k}=0$ and $\left(F^{2}\right)_{i j k}=0$. Since $F^{2}$ is locally a homogeneous function of degree 2 , it follows that $F^{2}=g_{i j}(x) y^{i} y^{j}$ for some $\left(g_{i j}(x)\right)$ depending only upon $x=\left(x^{i}\right)$. From the identity (5.31), we can see that the matrix $g_{i j}$ is nondegenerate (resp. positive definite) if and only if $\Pi$ is nondegenerate (resp. positive definite).

The local structure of $\mathscr{F}_{g}$ determines the Riemannian metric $g$ uniquely. More precisely, if $\mathscr{F}_{g}$ is equivalent to the Legendre foliation $\mathscr{F}_{\tilde{g}}$ obtained from a Riemannian manifold $(\widetilde{M}, \tilde{g})$, then $(M, g)$ and $(\widetilde{M}, \tilde{g})$ are isometric.

5.34 Proposition. Let $\phi: S^{*} M \rightarrow S^{*} \widetilde{M}$ be a diffeomorphism such that $\phi^{*} \eta_{\tilde{g}}=$ $\eta_{g}$ and $\phi^{*} \mathscr{F}_{\tilde{g}}=\mathscr{F}_{g}$. Then there is an isometry $\phi_{0}: M \rightarrow \widetilde{M}$ such that $\phi=$ $\left(\phi_{0}^{*}\right)^{-1} S^{*} M$.

Proof. The existence of $\phi_{0}$ with the property $\phi=\left(\phi_{0}^{*}\right)^{-1}$ follows from Corollary 2.6. Clearly, the map $\phi_{0}$ is an isometry since it preserves unit length (i.e. $\phi_{0}^{*}$ maps $S^{*} \widetilde{M}$ onto $S^{*} M$ ).

The local structure of a Riemannian Legendre foliation $\mathscr{F}_{g}$ can be explicitly described in terms of an orthonormal coframe as follows. Let $v=\left(v^{i}\right)$ be a local orthonormal coframe defined on an open set $U \subset M$, and let $\varphi=\left(\varphi_{j}^{i}\right)$ be 
the connection form for $v$. Then, the structure equations for the Riemannian structure are

$$
d v+\varphi \wedge v=0, \quad d \varphi+\varphi \wedge \varphi=\mathbf{\Omega}_{M}
$$

where $\boldsymbol{\Omega}_{M}=\left(\frac{1}{2} R_{j k l}^{i} v^{k} \wedge v^{l}\right)$ is the curvature matrix.

To define $\theta^{\alpha}, \xi^{\alpha}$, consider the parametrization of $T^{*} U$ defined by the coframe $v$ :

$$
\sum_{i=1}^{n+1} y^{i} v_{x}^{i} \Leftrightarrow\left(x, y^{1}, \ldots, y^{n+1}\right) \in U \times \mathbb{R}^{n+1} .
$$

Then the contact 1 -form can be written as $\eta=\sum_{i=1}^{n+1} y^{i} v^{i}$. We define $\theta^{\alpha}=f_{i}^{\alpha} v^{i}$ where $\mathbf{f}=\left(f_{j}^{i}\right)$ is chosen to be an $O(n+1, \mathbb{R})$-valued smooth function such that $f_{i}^{n+1}=y^{i}$ defined on an open subset of $S_{g}^{*} U$. Therefore, we have

$$
\Theta=\mathbf{f} v, \quad v=\mathbf{f}^{-1} \Theta, \quad \text { where } \Theta=\left(\begin{array}{c}
\theta^{\alpha} \\
\eta
\end{array}\right) .
$$

We define $\xi^{\alpha}$ as follows. By applying the exterior derivative to the first equation of (5.35), and using the identity $d \mathbf{f} \mathbf{f}^{-1}=-\mathbf{f} d \mathbf{f}^{-1}$,

$$
\begin{aligned}
d \boldsymbol{\Theta} & =d \mathbf{f} \wedge v+\mathbf{f} d v=d \mathbf{f} \wedge\left(\mathbf{f}^{-1} \boldsymbol{\Theta}\right)-\mathbf{f} \varphi \wedge\left(\mathbf{f}^{-1} \boldsymbol{\Theta}\right) \\
& =-\mathbf{f}\left(d \mathbf{f}^{-1}+\varphi \mathbf{f}^{-1}\right) \wedge \boldsymbol{\Theta} .
\end{aligned}
$$

Note that the matrix valued 1-form $\mathbf{f}\left(d \mathbf{f}^{-1}+\varphi \mathbf{f}^{-1}\right)$ is skew-symmetric. If we denote this by $\pi=\left(\pi_{j}^{i}\right)$ and let $\xi^{\alpha}=\pi_{\alpha}^{n+1}=-\pi_{n+1}^{\alpha}$, we have

$$
\left(\begin{array}{c}
d \theta^{\alpha} \\
d \eta
\end{array}\right)=-\left(\begin{array}{cc}
\pi_{\beta}^{\alpha} & -\xi^{\alpha} \\
\xi^{\beta} & 0
\end{array}\right)\left(\begin{array}{c}
\theta^{\beta} \\
\eta
\end{array}\right) \text {. }
$$

We claim that $\left(\theta^{\alpha}, \eta, \xi^{\alpha}\right)$ is a normalized coframe for $\mathscr{F}_{g}$, and $\pi_{\beta}^{\alpha}$ is the connection form for $\nabla$ defined in Theorem 5.10. In fact, this can be checked by using the following structure equations.

5.37 Proposition. The coframe $\theta^{\alpha}, \eta, \xi^{\alpha}$ satisfies the following structure equations:

$$
\begin{gathered}
\left(\begin{array}{c}
d \theta^{\alpha} \\
d \eta \\
d \xi^{\alpha}
\end{array}\right)=-\left(\begin{array}{ccc}
\pi_{\beta}^{\alpha} & 0 & 0 \\
0 & 0 & 0 \\
0 & 0 & \pi_{\beta}^{\alpha}
\end{array}\right)\left(\begin{array}{c}
\theta^{\beta} \\
\eta \\
\xi^{\beta}
\end{array}\right)+\left(\begin{array}{c}
-\delta_{\beta}^{\alpha} \eta \wedge \xi^{\beta} \\
\delta_{\beta \gamma} \theta^{\beta} \wedge \xi^{\gamma} \\
\left(-\mathbf{f} \boldsymbol{\Omega}_{M} \mathbf{f}^{-1}\right)_{n+1}^{\alpha}
\end{array}\right), \\
d \pi_{\beta}^{\alpha}+\pi_{\gamma}^{\alpha} \wedge \pi_{\beta}^{\gamma}=\xi^{\alpha} \wedge \xi^{\beta}+\left(\mathbf{f} \boldsymbol{\Omega}_{M} \mathbf{f}^{-1}\right)_{\beta}^{\alpha} .
\end{gathered}
$$

Proof. The first two equations in (5.38) are immediate consequences of (5.36). The rest of the equations follow from

$$
d \pi+\pi \wedge \pi=\mathbf{f} \mathbf{\Omega}_{M} \mathbf{f}^{-1} .
$$


To prove this, multiply $\mathbf{f}^{-1}$ on both sides of $\pi=\mathbf{f}\left(d \mathbf{f}^{-1}+\varphi \mathbf{f}^{-1}\right)$. Then we have $\mathbf{f}^{-1} \pi=d \mathbf{f}^{-1}+\varphi \mathbf{f}^{-1}$. By applying the exterior derivative on both sides of this,

$$
\begin{aligned}
d \mathbf{f}^{-1} \wedge \pi+\mathbf{f}^{-1} d \pi & =(d \varphi) \mathbf{f}^{-1}-\varphi \wedge\left(d \mathbf{f}^{-1}\right) \\
& =\left(-\varphi \wedge \varphi+\mathbf{\Omega}_{M}\right) \mathbf{f}^{-1}-\varphi \wedge\left(d \mathbf{f}^{-1}\right) \\
& =-\varphi \wedge\left(d \mathbf{f}^{-1}+\varphi \mathbf{f}^{-1}\right)+\boldsymbol{\Omega}_{M} \mathbf{f}^{-1} \\
& =-\left(\varphi \mathbf{f}^{-1}\right) \wedge \pi+\boldsymbol{\Omega}_{M} \mathbf{f}^{-1} .
\end{aligned}
$$

From this, we obtain

$$
\mathbf{f}^{-1} d \pi=-\left(d \mathbf{f}^{-1}+\varphi \mathbf{f}^{-1}\right) \wedge \pi+\boldsymbol{\Omega}_{M} \mathbf{f}^{-1}=-\mathbf{f}^{-1} \pi \wedge \pi+\boldsymbol{\Omega}_{M} \mathbf{f}^{-1},
$$

and (5.39) follows from this.

5.40 Corollary. Let $\mathscr{F}_{g}$ be the Riemannian Legendre foliation defined on $S_{g}^{*} M$, where $(M, g)$ is a Riemannian manifold. Then the metric has constant sectional curvature $\kappa$ if and only if $K_{\beta \gamma}^{\alpha}=0$ and $S_{\beta}^{\alpha}=2 \kappa \delta_{\beta}^{\alpha}$.

Proof. Comparing the equation (5.38) with the structure equation (5.25), it can be easily checked that $K_{\beta \gamma}^{\alpha}$ is the coefficient of $\theta^{\beta} \wedge \theta^{\gamma}$ and $S_{\beta}^{\alpha}$ is the coefficient of $\eta \wedge \theta^{\beta}$ in $\left(-\mathbf{f} \boldsymbol{\Omega}_{M} \mathbf{f}^{-1}\right)_{n+1}^{\alpha}$ where $\boldsymbol{\Omega}_{M}=\left(\frac{1}{2} R^{i}{ }_{j k l} v^{k} \wedge v^{l}\right)$ is the curvature matrix for the metric $g$.

If $g$ has constant sectional curvature $\kappa$, then $R_{j k l}^{i}=\kappa\left(\delta_{k}^{i} \delta_{l}^{j}-\delta_{l}^{i} \delta_{k}^{j}\right)$ and we have $\left(\mathbf{\Omega}_{M}\right)_{j}^{i}=\kappa v^{i} \wedge v^{j}$. From this and the identity $\mathbf{f}^{-1}=\mathbf{f}^{t}$, it follows that $\left(\mathbf{f} \boldsymbol{\Omega}_{M} \mathbf{f}^{-1}\right)_{n+1}^{\alpha}=\kappa(\mathbf{f} v)^{\alpha} \wedge(\mathbf{f} v)^{n+1}=\kappa \theta^{\alpha} \wedge \eta$. This in turn implies the equations $K_{\beta \gamma}^{\alpha}=0$ and $S_{\beta}^{\alpha}=\kappa \delta_{\beta}^{\alpha}$. since

Conversely, if $K_{\beta \gamma}^{\alpha}=0$ and $S_{\beta}^{\alpha}=\kappa \delta_{\beta}^{\alpha}$, then $\left(\mathbf{f} \boldsymbol{\Omega}_{M} \mathbf{f}^{-1}\right)_{n+1}^{\alpha}=\kappa \theta^{\alpha} \wedge \eta$. But,

$$
\begin{aligned}
\left(\mathbf{f} \boldsymbol{\Omega}_{M} \mathbf{f}^{-1}\right)_{n+1}^{\alpha} & =\frac{1}{2} f_{i}^{\alpha} R_{j k l}^{i} y^{j} v^{k} \wedge v^{l}, \quad \text { and } \\
2 \kappa \theta^{\alpha} \wedge \eta & =\sum_{j i} \kappa f_{i}^{\alpha} y^{j} v^{i} \wedge v^{j}=\sum_{j i} \kappa f_{i}^{\alpha}\left(\delta_{k}^{i} \delta_{l}^{j}-\delta_{k}^{j} \delta_{l}^{i}\right) y^{j} v^{k} \wedge v^{l} .
\end{aligned}
$$

From these, we obtain $f_{i}^{\alpha} R_{j k l}^{i} y^{j}=\kappa f_{i}^{\alpha}\left(\delta_{k}^{i} \delta_{l}^{j}-\delta_{k}^{j} \delta_{l}^{i}\right) y^{j}$. Note that this identity holds even when $\alpha$ is replaced by $n+1$ since both sides vanish. Moreover, since $R^{i}{ }_{j k l}$ and $\kappa$ do not depend on $y^{i}$, it follows that $R^{i}{ }_{j k l}=$ $\kappa\left(\delta_{k}^{i} \delta_{l}^{j}-\delta_{k}^{j} \delta_{l}^{i}\right)$.

5.41 Canonical CR-structures on $P$. A $C R$-structure on $P$ is an $n$-dimensional complex subbundle $\mathscr{H}$ of the complexified tangent bundle $\mathbb{C} T P$ of $P$ satisfying $\mathscr{H} \cap \overline{\mathscr{H}}=\{0\}$ where the bar denotes the complex conjugation. A CRstructure is called integrable if $[\mathscr{H}, \mathscr{H}] \subset \mathscr{H}$ (i.e. the set of smooth sections is closed under the Lie bracket). It can be easily checked that the integrability of the CR-structure is equivalent to condition that the ideal in the exterior algebra 
$\bigwedge\left(\mathbb{C} T^{*} P\right)$ generated by $\mathscr{H}^{\perp}=\operatorname{Ann}(\mathscr{H}) \subset \mathbb{C} T^{*} P$ is closed under the exterior differentiation.

If $\mathscr{F}$ is nondegenerate, there is a canonical CR-structure on $P$ defined by the bundle $\mathscr{H}$ spanned by $W_{\alpha}-i \varepsilon_{\alpha}^{\beta} X_{\beta}$.

5.42 Proposition. The CR-structure defined by $\mathscr{H}=\operatorname{span}\left(W_{\alpha}-i \varepsilon_{\alpha}^{\beta} X_{\beta}\right)_{\alpha=1 \cdots n}$ is integrable if and only if $K_{\beta \gamma}^{\alpha}=0$. In particular, this CR-structure is integrable if $\mathscr{F}$ is locally a Riemannian Legendre foliation obtained from a Riemannian manifold with constant curvature (see Corollary 5.40).

Proof. Let $\vartheta^{\alpha}=\frac{1}{2}\left(\theta^{\alpha}+i \varepsilon_{\beta}^{\alpha} \xi^{\beta}\right)$. Then the 1 -forms $\bar{\vartheta}^{\alpha}$ and $\eta$ span $\mathscr{H}^{\perp}$, and the integrability condition can be equivalently stated as

$$
d \eta \equiv d \bar{\vartheta}^{\alpha} \equiv 0 \quad \bmod \left(\eta, \bar{\vartheta}^{\alpha}\right) .
$$

To compute $d \bar{\vartheta}^{\alpha}$ and $d \eta$, note that the 1 -forms $\vartheta^{\alpha}, \theta^{\alpha}$, and $\xi^{\alpha}$ have the following relations:

$$
\theta^{\alpha} \equiv \vartheta^{\alpha}, \quad \xi^{\alpha}=-i \varepsilon_{\beta}^{\alpha} \vartheta^{\alpha} \quad \bmod \left(\eta, \bar{\vartheta}^{\alpha}\right)
$$

From these relations and the structure equation (5.21), we have $d \eta=\delta_{\alpha \beta} \theta^{\alpha} \wedge$ $\xi^{\beta} \equiv \varepsilon_{\alpha \beta} \vartheta^{\alpha} \wedge \bar{\vartheta}^{\beta} \equiv 0 \bmod \left(\eta, \bar{\vartheta}^{\alpha}\right)$.

Also, using the structure equation (5.21), we compute $d \bar{\vartheta}^{\alpha}$ as follows:

$$
\begin{aligned}
d \bar{\vartheta}^{\alpha}= & -\frac{1}{2}\left(d \theta^{\alpha}-i \varepsilon_{\beta}^{\alpha} d \xi^{\beta}\right) \\
= & \frac{1}{2}\left\{-\pi_{\beta}^{\alpha} \wedge \theta^{\beta}-\varepsilon_{\beta}^{\alpha} \eta \wedge \xi^{\beta}+G^{\alpha}{ }_{\sigma \gamma} \varepsilon_{\beta}^{\sigma} \theta^{\beta} \wedge \xi^{\gamma}\right\} \\
& -\frac{i}{2} \varepsilon_{\beta}^{\alpha}\left\{(\pi)_{\gamma}^{\beta} \wedge \xi^{\gamma}+K_{\gamma \sigma}^{\beta} \theta^{\gamma} \wedge \theta^{\sigma}+S_{\gamma}^{\beta} \eta \wedge \theta^{\gamma}+Q_{\gamma \sigma}^{\alpha} \theta^{\gamma} \wedge \xi^{\sigma}\right\} .
\end{aligned}
$$

Since $G_{\gamma \rho}^{\alpha}$ and $Q^{\beta}{ }_{\gamma \sigma} \varepsilon_{\rho}^{\sigma}$ are symmetric in $\gamma, \rho$, we have

$$
\begin{aligned}
G_{\sigma \gamma}^{\alpha} \varepsilon_{\beta}^{\sigma} \theta^{\beta} & \wedge \xi^{\gamma} \equiv-i G_{\sigma \gamma}^{\alpha} \varepsilon_{\beta}^{\sigma} \varepsilon_{\rho}^{\gamma} \vartheta^{\beta} \wedge \vartheta^{\rho} \equiv 0 \bmod \left(\eta, \bar{\vartheta}^{\alpha}\right), \\
Q^{\beta}{ }_{\gamma \sigma} \theta^{\gamma} & \wedge \xi^{\sigma} \equiv-Q_{\gamma \sigma}^{\beta}{ }_{\gamma \sigma}^{\sigma} \vartheta^{\sigma} \wedge \vartheta^{\rho} \equiv 0 \bmod \left(\eta, \bar{\vartheta}^{\alpha}\right) .
\end{aligned}
$$

Thus, from the equation (5.43), and the fact that $\varepsilon \pi^{t}=-\pi \varepsilon$ (recall that $\pi$ is an $o(p, q)$-valued 1-form),

$$
\begin{aligned}
d \bar{\vartheta}^{\alpha} & \equiv-\frac{1}{2} \pi_{\beta}^{\alpha} \wedge \theta^{\beta}+\frac{i}{2} \pi_{\beta}^{\alpha} \varepsilon_{\gamma}^{\beta} \xi^{\gamma}-\frac{i}{2} \varepsilon_{\beta}^{\alpha} K_{\gamma \sigma}^{\beta} \theta^{\gamma} \wedge \theta^{\sigma} \\
& \equiv-\pi_{\beta}^{\alpha} \wedge\left\{\frac{1}{2}\left(\theta^{\beta}-i \varepsilon_{\gamma}^{\beta} \xi^{\gamma}\right)\right\}-\frac{i}{2} \varepsilon_{\beta}^{\alpha} K_{\gamma \sigma}^{\beta} \vartheta^{\gamma} \wedge \vartheta^{\sigma} \\
& \equiv-\pi_{\beta}^{\alpha} \wedge \vartheta^{\beta}-\frac{i}{2} \varepsilon_{\beta}^{\alpha} K_{\gamma \sigma}^{\beta} \vartheta^{\gamma} \wedge \vartheta^{\sigma} \equiv-\frac{i}{2} \varepsilon_{\beta}^{\alpha} K_{\gamma \sigma}^{\beta} \vartheta^{\gamma} \wedge \theta^{\sigma}
\end{aligned}
$$

modulo $\left(\eta, \bar{\vartheta}^{\alpha}\right)$. From this, it follows that $K_{\beta \gamma}^{\alpha}=0$ if and only if $d \bar{\vartheta}^{\alpha} \equiv 0$ $\bmod \left(\eta, \bar{\vartheta}^{\alpha}\right)$ and the theorem is proved. 
In the special case when $\operatorname{dim} P=5$ and $\Pi$ is positive definite, a different CR-structure can be defined as follows: Observe that in this case the canonical reduction of structure group in Theorem 5.4 gives an $O(2, \mathbb{R})$-bundle of frames over $P$. By choosing an orientation of this bundle, and using the fact that $S O(2, \mathbb{R}) \cong U(1) \cong\left\{e^{i t} \in \mathbb{C} \mid t \in \mathbb{R}\right\}$, we define a complex structure $J: \mathbb{C} \operatorname{Ann}(\eta) \longrightarrow \mathbb{C A n n}(\eta)$ by the multiplication of $i$. More precisely, we define $J$ by the relations

$$
J W_{1}=W_{2}, \quad J W_{2}=-W_{1}, \quad J X_{1}=X_{2}, \quad \text { and } \quad J X_{2}=-X_{1},
$$

and extend by linearity. The CR-structure $\mathscr{H}$ on $P$ is defined by the $i$ eigenspace of $J$, or equivalently by the bundle spanned by $W_{1}-i W_{2}$ and $X_{1}-i X_{2}$.

5.45 Proposition. The CR-structure defined above is integrable if and only if

$$
G_{111}=3 G_{122} \text { and } G_{222}=3 G_{112} .
$$

Proof. Note that the 1-forms $\bar{\vartheta}^{1}=\frac{1}{2}\left(\theta^{1}-i \theta^{2}\right), \bar{\vartheta}^{2}=\frac{1}{2}\left(\xi^{1}-i \xi^{2}\right)$, and $\eta$ span $\mathscr{H}^{\perp}$. Hence, to prove the proposition, we need to show that the condition (5.46) are equivalent to $d \bar{\vartheta}^{1} \equiv d \bar{\vartheta}^{2} \equiv d \eta \equiv 0 \bmod \left(\eta, \bar{\vartheta}^{1}, \bar{\vartheta}^{2}\right)$. To show this, note that $\theta^{1} \equiv \vartheta^{1}, \theta^{2} \equiv-i \vartheta^{1}, \xi^{1} \equiv \vartheta^{2}$, and $\xi^{2} \equiv-i \vartheta^{2}$. Using the structure equation (5.21), and the fact that $\pi_{\beta}^{\alpha}$ and $K_{\alpha \beta}^{\sigma}$ are skew-symmetric in $\alpha, \beta$ the following can be shown by direct computation:

$$
\begin{aligned}
d \eta & =\theta^{1} \wedge \xi^{1}+\theta^{2} \wedge \xi^{2} \equiv \vartheta^{1} \wedge \vartheta^{2}-\vartheta^{1} \wedge \vartheta^{2} \equiv 0, \\
d \bar{\vartheta}^{1} & \equiv \frac{1}{2}\left\{\left(G_{11}^{1}-3 G_{22}^{1}\right)+i\left(G_{22}^{2}-3 G_{12}^{1}\right)\right\} \vartheta^{1} \wedge \vartheta^{2}, \\
d \bar{\vartheta}^{2} & \equiv \frac{1}{2}\left\{\left(Q_{11}^{1}-3 Q_{22}^{1}\right)+i\left(Q_{22}^{2}-3 Q_{12}^{1}\right)\right\} \vartheta^{1} \wedge \vartheta^{2}
\end{aligned}
$$

modulo $\left(\eta, \bar{\vartheta}^{1}, \bar{\vartheta}^{2}\right)$. From this, it follows that the condition $d \bar{\vartheta}^{1} \equiv d \bar{\vartheta}^{2} \equiv$ $d \eta \equiv 0$ implies the condition (5.46). To prove the converse, suppose that the condition (5.46) holds. Recall from Proposition 5.23 that $\nabla_{\mathbf{x}_{\eta}} \mathbf{G}=-Q_{\alpha \beta \gamma} \xi^{\alpha} \otimes$ $\xi^{\beta} \otimes \xi^{\gamma}$. Thus, the following identity holds:

$$
Q_{\alpha \beta \gamma}=-\mathbf{X}_{\eta}\left(G_{\alpha \beta \gamma}\right)+\pi_{\alpha}^{\rho}\left(\mathbf{X}_{\eta}\right) G_{\rho \beta \gamma}+\pi_{\beta}^{\rho}\left(\mathbf{X}_{\eta}\right) G_{\alpha \rho \gamma}+\pi_{\gamma}^{\rho}\left(\mathbf{X}_{\eta}\right) G_{\alpha \beta \rho} .
$$

It can be directly computed using this identity that $Q_{111}=3 Q_{122}$, and $Q_{222}=$ $3 Q_{112}$, and the condition $d \bar{\vartheta}^{1} \equiv d \bar{\vartheta}^{2} \equiv d \eta \equiv 0$ follows from the identities (5.47) and (5.48).

In the special case when $\mathscr{F}$ is the Riemannian Legendre foliation defined on the unit cotangent bundle of a Riemannian manifold, this CR-structure coincides with the one defined by LeBrun [LB]. In this case, the conditions (5.46) is trivially satisfied because the invariant $\mathbf{G}$ vanishes, and thus the CR-structure is integrable by the previous proposition. 


\section{Global Results}

In this section, we study the topology of Legendre foliations. The fundamental result of this section is that every simply connected and compact leaf of a Legendre foliation $\mathscr{F}$ is diffeomorphic to a sphere. If all the leaves of $\mathscr{F}$ are compact and simply connected, $\mathscr{F}$ is globally equivalent to one of the form $\mathscr{F}_{F}$ defined on a unit cotangent bundle with all fibers diffeomorphic to the spheres. Under the additional condition that $\Pi$ is nondegenerate, $F$ is a Finsler metric.

6.1 Compact and simply connected leaves. To prove this, recall from the normal form theorem that there is an immersion $\phi_{\eta}: V \rightarrow T^{*}(V / \mathscr{F})$ where $V$ is a neighborhood of a point in $P$ such that the leaf space $V / \mathscr{F}$ of $V$ is a manifold. If the foliation $\mathscr{F}$ defines a quotient manifold over $P$, the map $\phi_{\eta}$ can be extended to $P$ by the same construction:

$$
\phi_{\eta}: P \rightarrow T^{*}(P / \mathscr{F}) .
$$

and each $\Sigma \in \mathscr{F}$ is immersed into a fiber of $T^{*}(P / \mathscr{F})$ transversal to radial lines under $\phi_{\eta}$.

From the theory of foliations, it can be shown that if $\Sigma \in \mathscr{F}$ is a compact and simply connected leaf, then there is a neighborhood $V$ of $\Sigma$ over which the foliation restricted to $V$ defines a quotient manifold [L]. Consequently, if all the leaves are simply connected and compact, $P / \mathscr{F}$ is a manifold.

6.2 Theorem. A compact and simply connected leaf of a Legendre foliation $\mathscr{F}$ is diffeomorphic to a sphere.

Proof. The case $n=1$ is trivial. When $n>1$, consider the map $\phi_{\eta}: V \rightarrow$ $T^{*}(V / \mathscr{F})$ defined as above. The map $\phi_{\eta}$ maps $\Sigma$ into $T_{x}^{*}(V / \mathscr{F})-\{0\}$ for some $x \in(V / \mathscr{F})$. Consider a unit cotangent bundle $S^{*}(V / \mathscr{F}) \subset T^{*}(V / \mathscr{F})$ with respect to some metric $g$ on $V / \mathscr{F}$. If we denote $\rho$ for the radial projection from $T_{0}^{*}(V / \mathscr{F})$ to $S^{*}(V / \mathscr{F})$, the composition map

$$
\left(\rho \circ \phi_{\eta}\right)_{\mid \Sigma}: \Sigma \rightarrow S_{x}^{*}(V / \mathscr{F})
$$

is a local diffeomorphism since the map $\left(\phi_{\eta}\right)_{\mid \Sigma}: \Sigma \rightarrow T_{x}^{*}(V / \mathscr{F})$ is transversal to the radial lines. Since $\Sigma$ is compact, the map $\left(\rho \circ \phi_{\eta}\right)_{\mid \Sigma}$ is onto, and hence it is a covering map of the sphere. From this, it follows that $\left(\rho \circ \phi_{\eta}\right)_{\mid \Sigma}$ is a diffeomorphism since $S_{x}^{*}(V / \mathscr{F})$ is simply connected.

Under the condition that all the leaves are compact and simply connected, the normal form theorem generalizes to a global theorem.

6.3 Corollary. If all the leaves of $\mathscr{F}$ are compact and simply connected, $\mathscr{F}$ is globally equivalent to a Legendre foliation of the form $\mathscr{F}_{F}$. Moreover, if $\mathscr{F}$ is nondegenerate, $F$ is a Finsler metric.

Proof. Since all the leaves are simply connected and compact, the leaf space $M=P / \mathscr{F}$ of $\mathscr{F}$ is a manifold [L]. Thus, we can take $V=P$ and obtain the 
map $\phi_{\eta}: P \rightarrow T^{*} M$ as in the proof of the previous theorem. It is clear that $\phi_{\eta}$ is injective since $\rho \circ \phi_{\eta}$ is injective. Moreover, $\phi_{\eta}$ is an embedding of $P$ into $T^{*} M$ since $\phi_{\eta}=\psi \circ \eta$ where $\psi$ is a local diffeomorphism and $\eta$ is an embedding. By defining $S_{F}^{*} M=\phi_{\eta}(P)$, we obtain the foliation $\mathscr{F}_{F}$. (See the proof of the normal form theorem.)

We now show that, under the additional assumption that $\mathscr{F}$ is nondegenerate $F$ is a Finsler metric by showing that $\left(F^{2}\right)_{i j}$ is positive definite (see the identity (5.31)). But, since fibers of $S_{F}^{*} M$ are connected, it suffices to show this at some point in $v_{0} \in T_{x}^{*} M$ for each $x \in M$. We claim that if we take $v_{0} \in T_{x}^{*} M$ such that $\left\|v_{0}\right\|=\sup \left\{\|v\| F(v)=1, v \in T_{x}^{*} M\right\}$ where $\|v\|=\sqrt{\sum_{i=1}^{n+1}\left(y^{i}\right)^{2}}$, then $\left(F^{2}\right)_{i j}\left(v_{0}\right)$ is positive definite. To show this, let $\left(u^{i}\right) \in \mathbb{R}^{n+1}$, and choose a curve $\gamma: \mathbb{R} \rightarrow T_{x}^{*} M$ tangent to $\mathscr{F}_{F}$ such that $\gamma(0)=v_{0}$ and $\frac{d}{d t} \gamma_{\mid t=0}=u^{i} \frac{\partial}{\partial y^{i}}$. Then, if we let $a(t)=\left\|v_{0}\right\|^{2} F^{2}(\gamma(t))-\|\gamma(t)\|^{2}$, then $a(0)=0$ and $a(v) \geq 0$ for $v \in T_{x}^{*} M$. Thus $a(t)$ has a local minimum at 0 , and $\frac{d^{2} a}{d t^{2}}(0) \geq 0$. Therefore, we have

$$
\left\|v_{0}\right\|^{2}\left(F^{2}\right)_{i j} u^{i} u^{j}-\delta_{i j} u^{i} u^{j} \geq 0
$$

at $v_{0}$, and from this, it follows that $\left(F^{2}\right)_{i j}\left(v_{0}\right) u^{i} u^{j} \geq 0$.

Theorem 6.2 can be further generalized to the case when a compact leaf has finite fundamental group.

6.4 Theorem. A compact leaf of $\mathscr{F}$ with finite fundamental group is diffeomorphic to a quotient of a sphere.

The proof of this makes use of the following theeorem [L]:

Reeb Stability Theorem. Suppose that $\Sigma$ is a compact leaf of the foliation $\mathscr{F}$, and $\mathbf{p}: \widetilde{\Sigma} \rightarrow \Sigma$ is the universal covering map. If the fundamental group of $\Sigma$ is finite, then there is a neighborhood $V$ of $\Sigma$, and a finite covering map

$$
\tilde{\mathbf{p}}: B^{n+1} \times \widetilde{\Sigma} \rightarrow V
$$

such that $\tilde{\mathbf{p}}_{\mid\{0\} \times \widetilde{\Sigma}}=\mathbf{p}$, and $\tilde{\mathbf{p}}^{*}(\mathscr{F})$ is the foliation defined by fibers of the projection map $\pi: B^{n+1} \times \widetilde{\Sigma} \rightarrow B^{n+1}$ defined by $\pi(x, y)=x$, where $B^{n+1}$ is a open unit ball.

Proof of Theorem 6.4. On $B^{n+1} \times \tilde{\Sigma}$, we define a contact structure and Legendre foliation by $\tilde{\mathbf{p}}^{*} \eta$ and $\tilde{\mathbf{p}}^{*} \mathscr{F}$. Since $\Sigma$ has finite fundamental group and compact, $\widetilde{\Sigma}$ is compact leaf of $\tilde{\mathbf{p}}^{*} \mathscr{F}$. From Theorem 6.2 , it follows that $\widetilde{\Sigma}$ is diffeomorphic to the sphere.

The following example is the construction of a compact leaf diffeomorphic to the quotient of a sphere from the unit cotangent bundle of a Riemannian manifold. 
Example 6.5. Let $G$ be a finite subgroup of $O(n+1, \mathbb{R})$ acting on $\mathbb{R}^{n+1}$. Also, we assume that all the elements, except the identity $I$, has only one fixed point 0 . For example, we can take $G=\{ \pm I\} \subseteq O(n+1, \mathbb{R})$. Since each element of $G$ preserves the canonical flat metric on $\mathbb{R}^{n+1}$, we can define a right group action of $G$ on $S^{*} \mathbb{R}^{n+1}$ by $v \cdot A=A^{*}(v)$ where $v \in S^{*} \mathbb{R}^{n+1}, A \in G$, and $A^{*}: T^{*} \mathbb{R}^{n+1} \longrightarrow T^{*} \mathbb{R}^{n+1}$ is the pull back of the derivative $A_{*}$ of the map $A: \mathbb{R}^{n+1} \rightarrow \mathbb{R}^{n+1}$. With respect to the standard coordinates on $T^{*} \mathbb{R}^{n+1}$, we express this group action as

$$
\left(x^{i}, y^{j}\right) \cdot A=\left(A_{k}^{i} x^{k}, A_{k}^{j} y^{k}\right), \quad \text { where }\left(x^{i}, y^{j}\right) \in S^{*} \mathbb{R}^{n+1} \subseteq T^{*} \mathbb{R}^{n+1} .
$$

From this and the assumption that $A \neq I$ has no fixed point other than 0 , it follows that $A$ has no fixed point on $S^{*} \mathbb{R}^{n+1}$. Therefore, since $G$ is finite, we can define a quotient manifold $P=S^{*} \mathbb{R}^{n+1} / G$ of $S^{*} \mathbb{R}^{n+1}$ with the natural projection $\pi_{G}: S^{*} \mathbb{R}^{n+1} \rightarrow S^{*} \mathbb{R}^{n+1} / G$. Note that, since $A^{*}: S^{*} \mathbb{R}^{n+1} \rightarrow S^{*} \mathbb{R}^{n+1}$ preserves the contact 1-form and fibers of $S^{*} \mathbb{R}^{n+1}$, one can define the contact 1 -form and the Legendre foliation $\mathscr{F}$ on $P$ induced from the contact 1 -form and fibers of $S^{*} \mathbb{R}^{n+1}$.

Observe that $0 \in \mathbb{R}^{n+1}$ is the only fixed point under the action of elements of $G$ different from I. Thus, $S_{0}^{*} \mathbb{R}^{n+1}=\pi^{-1}(0)$ is diffeomorphically mapped onto itself under the action of $A$. If we let $\Sigma_{0}=\pi_{G}\left(S_{0}^{*} \mathbb{R}^{n+1}\right)$, then $\Sigma_{0}$ is a compact leaf of $\mathscr{F}$ diffeomorphic to a quotient of a sphere. In the special case is when $G=\{ \pm I\}$, we have $\Sigma_{0}=\mathbb{R}^{n}$.

However, not every compact leaf of a Legendre foliation is diffeomorphic to the quotient of a sphere, as the following example shows:

Example 6.6. Consider the Legendre foliation on $S_{F}^{*} \mathbb{R}^{n+1}$ with $F$ defined by $F=y^{n+1}$. Then, the contact 1 -form can be written as

$$
\eta=d x^{n+1}+\sum_{\alpha=1}^{n} y^{\alpha} d x^{\alpha},
$$

and $\left(x^{i}, y^{\alpha}\right)$ are coordinates on $S_{F}^{*} \mathbb{R}^{n+1}$. Now, we consider the subgroup $G \subseteq G l(n+1, \mathbb{R})$ defined by

$$
G=\left\{\left(\begin{array}{cc}
I_{n} & 0 \\
-s & 1
\end{array}\right) \mid s \in \mathbb{Z}^{n}\right\} \cong \mathbb{Z}^{n} .
$$

The group $G$ acts on $\mathbb{R}^{n+1}$ as matrix multiplication on the left. As in the previous example, we obtain a right group action of $G$ on $S_{F}^{*} \mathbb{R}^{n+1}$ defined by $v . A=A^{*}(v)$. Clearly the fibers are mapped onto fibers under this action. We claim that $\eta$ remains fixed. To see this, write the group action in terms of coordinates:

$$
\left(\begin{array}{c}
x^{\alpha} \\
x^{n+1} \\
y^{\alpha}
\end{array}\right) \cdot A=\left(\begin{array}{c}
x^{\alpha} \\
-s_{\beta} x^{\beta}+x^{n+1} \\
y^{\alpha}+s_{\alpha}
\end{array}\right) \equiv\left(\begin{array}{c}
\bar{x}^{\alpha} \\
\bar{x}^{n+1} \\
\bar{y}^{\alpha}
\end{array}\right) .
$$


The claim follows from the following computation:

$$
\begin{aligned}
d \bar{x}^{n+1}+\sum_{\alpha=1}^{n} \bar{y}^{\alpha} d \bar{x}^{\alpha} & =\left(d x^{n+1}-s_{\alpha} d x^{\alpha}\right)+\sum_{\alpha=1}^{n}\left(y^{\alpha}+s_{\alpha}\right) d x^{\alpha} \\
& =d x^{n+1}+\sum_{\alpha=1}^{n} y^{\alpha} d x^{\alpha} .
\end{aligned}
$$

It is not difficult to see that, if $A \in G$ and $A \neq I, A$ has no fixed point. Moreover, this group action on $S_{F}^{*} \mathbb{R}^{n+1}$ is discrete. Hence, following the same argument as in the previous example, a quotient space $P=S_{F}^{*} \mathbb{R}^{n+1} / G$ by $G$ is a contact manifold with Legendre foliation denoted by $\mathscr{F}$. Note that the fiber $S_{0}^{*} \mathbb{R}^{n+1}=\pi^{-1}(0)$ of $S_{F}^{*} \mathbb{R}^{n+1}$ at $0 \in \mathbb{R}^{n+1}$ is mapped diffeomorphically onto itself under the action of $G$, and thus, we have an action of $G$ on $S_{0}^{*} \mathbb{R}^{n+1}$. If we let $\Sigma_{0}$ denote the quotient manifold of $S_{0}^{*} \mathbb{R}^{n+1}$ under this action, $\Sigma_{0}$ is a compact leaf of $\mathscr{F}$ diffeomorphic to a torus. To see this, note that $G$ acts on $S_{0}^{*} \mathbb{R}^{n+1}$ as follows:

$$
\left(\begin{array}{c}
0 \\
0 \\
y^{\alpha}
\end{array}\right) \stackrel{A}{\longmapsto}\left(\begin{array}{c}
0 \\
0 \\
y^{\alpha}+s_{\alpha}
\end{array}\right)
$$

6.7 Riemannian Legendre foliations. Recall from Proposition 5.32 that $\mathscr{F}$ is Riemannian if and only if $\Pi$ is positive definite and $\mathbf{G}=0$. In this case, the local structure of $\mathscr{F}$ restricts the topology of its leaves: A compact leaf of $\mathscr{F}$ is diffeomorphic to a quotient of a sphere. Moreover, if $P$ is compact, then all the leaves of $\mathscr{F}$ are necessarily compact, and consequently, diffeomorphic to quotients of spheres.

6.8 Theorem. A compact leaf of a Riemannian Legendre foliation is diffeomorphic to a quotient of a sphere by a finite group.

Proof. Recall from Proposition 5.24 that if $\mathscr{F}$ is Riemannian Legendre foliation, this canonical metric on leaves has constant sectional curvature, and if a leaf of $\mathscr{F}$ is compact, the canonical Riemannian metric on leaves is complete. It is well known that a complete Riemannian manifold with positive constant sectional curvature is a quotient of a sphere by a finite group [Wo]. From this, the theorem follows.

Recall from the structure of nondegenerate Legendre foliations that there is a canonical connection $\nabla$ on $P$ (Theorem 5.10).

6.9 Theorem. Let $\mathscr{F}$ be a Riemannian Legendre foliation. If $\nabla$ is complete on $P$, the leaves of $\mathscr{F}$ are diffeomorphic to quotients of spheres by finite groups. In particular, if $P$ is compact, then $\nabla$ is complete and all the leaves are necessarily compact and hence are quotients of spheres.

Proof. To show that the leaves are diffeomorphic to quotients of spheres, we prove that the canonical Riemannian metric on leaves of $\mathscr{F}$ is complete. To 
do this, suppose that $\gamma:(-\delta, \delta) \rightarrow \Sigma$ is a geodesic on a leaf $\Sigma \in \mathscr{F}$ for $\delta>0$ with respect to the canonical Riemannian metric on $\Sigma$. We claim that $\gamma$ can be extended to $\mathbb{R}$. Recall that the Levi-Civita connection of the canonical Riemannian metric on $\Sigma$ is the restriction of $\nabla$ to $\Sigma$. (See 5.25.) Hence, $\gamma$ is also a geodesic of $\nabla$ in $P$. But since $\nabla$ is complete, $\gamma$ can be extended to $\mathbb{R}$ as a geodesic of $\nabla$ on $P$. Thus, we only need to prove that $\gamma(t) \in \Sigma$ for all $t \in \mathbb{R}$.

But this can be seen by the following fact: A submanifold $\Sigma \subset P$ is called auto-parallel if, for vector fields $X, Y$ on $\Sigma, \nabla_{X} Y$ is tangent to $\Sigma$. In [KN], it is proved that, if $\gamma$ is a geodesic of an auto-parallel submanifold $\Sigma$ with $\dot{\gamma}(0)$ tangent to $\Sigma$, then $\gamma(t) \in \Sigma$ for small $t$.

The assumption that $\mathscr{F}$ is Riemannian is essential in Theorem 6.8, for in the following example, we show that Theorem 6.8 does not hold under the weaker condition $\mathbf{G} \neq 0$ by constructing a compact leaf diffeomorphic to a quotient of $S^{n-1} \times \mathbb{R}$.

Example 6.10. Consider the function $F: T^{*} \mathbb{R}^{n+1} \rightarrow \mathbb{R}^{+} \cup\{0\}$ defined by

$$
F=\frac{\sum_{a=1}^{n}\left(y^{\alpha}\right)^{2}}{y^{n+1}}
$$

where $\left(x^{i}, y^{i}\right)$ are the canonical coordinates on $T^{*} \mathbb{R}^{n+1}$. Thus leaves of the Legendre foliation $\mathscr{F}_{F}$ are hypersurfaces in each fiber of $T^{*} \mathbb{R}^{n+1}$ defined by the equation $y^{n+1}=\sum_{\alpha=1}^{n}\left(y^{\alpha}\right)^{2}$, and $\left(y^{i}\right) \neq 0$. It is not difficult to see that the leaf of the Legendre foliation is diffeomorphic to $S^{n-1} \times \mathbb{R}$, and hence, it is simply connected for $n \geq 2$

We claim that $\Pi$ is positive definite. Recall that we have $\Pi=F_{i j} d y^{i} \otimes d y^{i}$ restricted to $L$. The derivatives of $\mathrm{F}$ on $S_{F}^{*} \mathbb{R}^{n+1}$ are

$$
\begin{gathered}
F_{\alpha}=\frac{2 y^{\alpha}}{y^{n+1}}, \quad F_{n+1}=-\frac{1}{y^{n+1}}, \quad F_{\alpha \beta}=\frac{2 \delta_{\alpha \beta}}{y^{n+1}}, \\
F_{\alpha, n+1}=\frac{-2 y^{\alpha}}{\left(y^{n+1}\right)^{2}}, \quad F_{n+1, n+1}=\frac{2}{\left(y^{n+1}\right)^{2}} .
\end{gathered}
$$

If $X=a^{i} \frac{\partial}{\partial y^{i}} \neq 0$ is a tangential vector field to $\mathscr{F}_{F}$ (i.e. $d F(X)=a^{i} F_{i}=0$ ), we have

$$
\Pi(X, X)=F_{i j} a^{i} a^{j}=2 \sum_{\alpha=1}^{n} \frac{\left(a^{\alpha}\right)^{2}}{y^{n+1}}+\frac{2 a^{n+1}}{\left(y^{n+1}\right)^{2}}\left\{\left(a^{n+1}\right)-2\left(\sum_{\alpha=1}^{n} y^{\alpha} a^{\alpha}\right)\right\} .
$$

But, since $a^{i} F_{i}=0$ and from (6.11), we get $a^{n+1}=2 \sum_{\alpha=1}^{n} y^{\alpha} a^{\alpha}$. Thus, we have

$$
\Pi(X, X)=F_{i j} a^{i} a^{j}=2 \sum_{\alpha=1}^{n} \frac{\left(a^{\alpha}\right)^{2}}{y^{n+1}}>0 .
$$


Now, as in the previous examples, we define a quotient manifold of $S^{*} \mathbb{R}^{n+1}$ under a discrete group action defined by

$$
\left(x^{i}, y^{j}\right) \stackrel{A}{\longrightarrow}\left(\left(A^{-1}\right)_{k}^{i} x^{k}, A_{k}^{j} y^{k}\right)
$$

where $A=\left(A_{j}^{i}\right)$ is an element of the group

$$
\left\{\left(\begin{array}{cc}
\frac{1}{2^{k}} I & 0 \\
0 & \frac{1}{4^{k}}
\end{array}\right) \mid \begin{array}{cl}
I: n \times n & \text { identity matrix } \\
k \in \mathbb{Z}
\end{array}\right\} \cong \mathbb{Z} .
$$

If $A=\left(\begin{array}{cc}\frac{1}{2^{k}} I & 0 \\ 0 & \frac{1}{4^{k}}\end{array}\right)$, then $A$ acts on $T^{*} \mathbb{R}^{n+1}$ as follows:

$$
\left(x^{\alpha}, x^{n+1}, y^{\alpha}, y^{n+1}\right) \stackrel{A}{\longrightarrow}\left(2^{k} x^{\alpha}, 4^{k} x^{n+1}, \frac{1}{2^{k}} y^{\alpha}, \frac{1}{4^{k}} y^{n+1}\right) .
$$

It is clear that $\mathbb{Z}$ acts on $S^{*} \mathbb{R}^{n+1}$ with no fixed points. It can be also directly checked that the group action preserves the contact 1 -form and the Legendre foliation. Thus, we can define Legendre foliation $\mathscr{F}$ on the quotient space $P=$ $S^{*} \mathbb{R}^{n+1} / \mathbb{Z}$. Observe that the fiber $S_{0}^{*} \mathbb{R}^{n+1}=\pi^{-1}(0) \cong S^{n-1} \times \mathbb{R}$ is an invariant subspace and that the orbit space $S_{0}^{*} \mathbb{R}^{n+1} / \mathbb{Z}$ is obviously not a quotient of a sphere.

\section{Degenerate LegendRe Foliations}

There is a large class of Legendre foliations with the rank of $\Pi$ is between 1 and $n-1$. We call Legendre foliations of this class degenerate. A complete classification of degenerate Legendre foliations is an unsolved problem. However, under the assumption that the rank of the null space $L_{0}$ of $\Pi$ is constant, it can be shown that $L_{0}$ is an involutive distribution and by the Frobenius theorem, $L_{0}$ defines a foliation $\mathscr{F}_{0}$. Observe that, since $L_{0} \subset L$, each leaf of $\mathscr{F}$ is foliated by leaves of $\mathscr{F}_{0}$. We show that the leaves of $\mathscr{F}_{0}$ have a natural flat affine structure.

7.1 Proposition. If $L_{0}=\{X \in L \mid \Pi(X, Y)=0$ for all $Y \in L\}$ and $\operatorname{rank}(L)$ is constant, then $L_{0}$ is an involutive distribution.

Proof. We show that $\Gamma\left(L_{0}\right)$ is closed under the Lie bracket. To do this, we need to show that if $X, X^{\prime} \in \Gamma\left(L_{0}\right)$, then $\Pi\left(\left[X, X^{\prime}\right], Y\right)=0$ for all $Y \in \Gamma(L)$. Using the definition of $\Pi$ and the fact that the curvature of the Bott connection vanishes, we compute

$$
\begin{aligned}
\Pi\left(\left[X, X^{\prime}\right], Y\right) & =-\left\{\nabla_{\left[X, X^{\prime}\right]}^{N} \nabla_{Y}^{N} \eta\right\}\left(\mathbf{X}_{\eta}\right) \\
& =-\left\{\nabla_{X}^{N} \nabla_{X^{\prime}}^{N} \nabla_{Y}^{N} \eta-\nabla_{X^{\prime}}^{N} \nabla_{X}^{N} \nabla_{Y}^{N} \eta\right\}\left(\mathbf{X}_{\eta}\right) .
\end{aligned}
$$

But since $\left\{\nabla_{X^{\prime}}^{N} \nabla_{Y}^{N} \eta\right\}\left(\mathbf{X}_{\eta}\right)=-\Pi\left(X^{\prime}, Y\right)=0$, it follows that $\nabla_{X^{\prime}}^{N} \nabla_{Y}^{N} \eta \in \Gamma\left(Q^{*}\right)$. Therefore, $\nabla_{X^{\prime}}^{N} \nabla_{Y}^{N} \eta=\Phi(Z)\left(=\nabla_{Z}^{N} \eta\right)$ for some $Z \in \Gamma(L)$. Similarly, there 
exists $Z^{\prime} \in \Gamma(L)$ such that $\nabla_{X}^{N} \nabla_{Y}^{N} \eta=\nabla_{Z^{\prime}}^{N}$. Using these, we rewrite the identity (7.2):

$$
\boldsymbol{\Pi}\left(\left[X, X^{\prime}\right], Y\right)=-\left\{\nabla_{X}^{N} \nabla_{Z}^{N} \eta-\nabla_{X^{\prime}}^{N} \nabla_{Z^{\prime}}^{N} \eta\right\}\left(\mathbf{X}_{\eta}\right)=0 .
$$

7.3 Proposition. For $X \in \Gamma\left(L_{0}\right), \Gamma\left(L_{0}\right) \subset \Gamma(L)$ is invariant under the map $\nabla_{X}^{L}: \Gamma(L) \rightarrow \Gamma(L)$. Moreover, the restriction of $\nabla_{X}^{L}$ to $L_{0}$ defines a flat torsion free partial connection on $L_{0}$.

Proof. We want to show that, if $Y \in \Gamma\left(L_{0}\right)$, then $\nabla_{X}^{L} Y \in \Gamma\left(L_{0}\right)$. Recall that $\nabla_{X}^{L} Y$ is defined by $\nabla_{X}^{L} Y=\Phi^{-1} \nabla_{X}^{Q} \nabla_{Y}^{N} \eta$. Furthermore, we have $\nabla_{X}^{Q} \nabla_{Y}^{N} \eta=$ $\nabla_{X}^{N} \nabla_{Y}^{N} \eta$ since $\left\{\nabla_{X}^{N} \nabla_{Y}^{N} \eta\right\}\left(\mathbf{X}_{\eta}\right)=-\Pi(X, Y)=0$ implies $\nabla_{X}^{N} \nabla_{Y}^{N} \eta \in \Gamma\left(Q^{*}\right)$. For simplicity, let us denote $W=\Phi^{-1}\left(\nabla_{X}^{N} \nabla_{Y}^{N} \eta\right)$.

We claim that $\Pi(Z, W)=0$ for all $Z \in \Gamma(L)$ (i.e. $\nabla_{X}^{L} Y \in \Gamma\left(L_{0}\right)$ ). Recall that $\nabla_{W}^{N}=\Phi(W)$. Again, using the fact that the curvature of $\nabla^{N}$ vanishes, we compute

$$
\begin{aligned}
\Pi(Z, W) & =-\left\{\nabla_{Z}^{N} \nabla_{W}^{N}\right\}\left(\mathbf{X}_{\eta}\right)=-\left\{\nabla_{Z}^{N} \nabla_{X}^{N} \nabla_{Y}^{N} \eta\right\}\left(\mathbf{X}_{\eta}\right) \\
& =-\left\{\nabla_{X}^{N} \nabla_{Z}^{N} \nabla_{Y}^{N} \eta+\nabla_{[Z, X]}^{N} \nabla_{Y}^{N} \eta\right\}\left(\mathbf{X}_{\eta}\right) \\
& =-\left\{\nabla_{X}^{N} \nabla_{Z}^{N} \nabla_{Y}^{N} \eta\right\}\left(\mathbf{X}_{\eta}\right)-\boldsymbol{\Pi}([Z, X], Y) .
\end{aligned}
$$

But, since $Y \in \Gamma\left(L_{0}\right), \Pi([Z, X], Y)=0$. Moreover, since

$$
\left\{\nabla_{Z}^{N} \nabla_{Y}^{N} \eta\right\}\left(\mathbf{X}_{\eta}\right)=-\Pi(Z, Y)=0,
$$

we have $\nabla_{Z}^{N} \nabla_{Y}^{N} \eta \in \Gamma\left(Q^{*}\right)$. This implies that $\nabla_{Z}^{N} \nabla_{Y}^{N} \eta=\nabla_{Y^{\prime}}^{N} \eta$ for some $Y^{\prime} \in$ $\Gamma(L)$ and hence

$$
\left\{\nabla_{X}^{N} \nabla_{Z}^{N} \nabla_{Y}^{N} \eta\right\}\left(\mathbf{X}_{\eta}\right)=\left\{\nabla_{X}^{N} \nabla_{Y^{\prime}}^{N} \eta\right\}\left(\mathbf{X}_{\eta}\right)=\Pi\left(X, Y^{\prime}\right)=0 .
$$

The fact that this connection on $L_{0}$ is flat and torsion free follows from Proposition 3.23: For $X, Y$, and $Z \in \Gamma\left(L_{0}\right)$,

$$
R^{L}(X, Y) Z=\Pi(X, Z) Y-\Pi(Y, Z) X=0 .
$$

Recall that the partial connection $\nabla^{L}$ induces an ordinary affine connection on leaves of $\mathscr{F}$. Similarly, leaves of $\mathscr{F}_{0}$ possess a canonical affine connection induced from $\nabla^{L}$. Clearly, from the Proposition 7.3, this affine connection is flat and torsion free. Hence we have the corollary:

7.5 Corollary. Leaves of $\mathscr{F}_{0}$ have a natural flat affine structure.

\section{REFERENCES}

[B] D. E. Blair, Contact manifolds in Riemannian geometry, Lecture Notes in Math., vol. 509, Springer, Berlin, Heidelberg, and New York, 1976, pp. 36-37.

[BGJ] R. Bott, S. Gitler, and I. James, Lectures on algebraic and differential topology, Lecture Notes in Math., vol. 279, Springer, Berlin, Heidelberg, and New York, 1972.

[Br] R. L. Bryant, On notions of equivalence of variational problems with one independent variable, Contemp. Math., vol. 68, Amer. Math. Soc., Providence, R. I., 1987, pp. 65-67. 
[C] S. S. Chern, Local equivalence and Euclidean connection in Finsler spaces, Sci. Rep. Tsing Hua Univ. 5 (1948), 95-121.

[DK] T. Duchamp and Morris Kalka, Invariants of complex foliations and the Monge-Ampere equation.

[DPU] T. Duchamp, M. Pang and G. Uhlmann, Inverse scattering for metrics (work in progress).

[G] R. B. Gardner, Differential geometric methods interfacing control theory, Differential Geometric Control Theory. (R. Millman, H. Sussman, Eds.), Progress in Math., 27, Birkhäuser, Boston, Mass., 1983, pp. 117-180.

[KN] S. Kobayashi and K. Nomizu, Foundations of differential geometry, 2, Interscience, New York, pp. 53-61.

[KO] N. Kamran and P. Olver, The equivalence problem for particle Lagrangians, J. Differential Equations (to appear).

[KT] F. Kamber, Ph. Tondeur, Foliated bundles and characteristic classes, Lecture Notes in Math., vol. 493, Springer, Berlin, Heidelberg, and New York, 1975.

[L] H. Blaine Lawson, Jr., The quantitative theory of foliations, C.B.M.S. Regional Conf. Ser. in Math., no. 27, Amer. Math. Soc., Providence, R. I., 1977.

[LB] C. R. LeBrun, Twister CR manifolds and three-dimensional conformal geometry, Trans. Amer. Math. Soc. 284 (1984), 601-616.

[Sp] M. Spivak, A comprehensive introduction to differential geometry, vol. 2, Publish or Perish, pp. 205-216.

[St] S. Sternberg, Lectures on differential geometry, Prentice-Hall, Englwood Cliffs, N. J., 1964.

[W] A. Weinstein, Symplectic manifolds and their Lagrangian submanifolds, Adv. in Math. 6 (1971), 329-346.

[Wo] J. A. Wolf, Spaces of constant curvature, Publish or Perish.

Department of Mathematics, University of Washington, Seattle, Washington 98195 